Check for updates

Cite this: RSC Adv., 2017, 7, 53785

Received 1st August 2017

Accepted 2nd November 2017

DOI: $10.1039 / c 7 r a 08499 d$

rsc.li/rsc-advances

\section{Polyfluorenylacetylene for near-infrared laser protection: polymer synthesis, optical limiting mechanism and relationship between molecular structure and properties $\dagger$}

\author{
Gang Zhao, ${ }^{a}$ Yan Feng, ${ }^{b}$ Shanyi Guang, ${ }^{\star c}$ Hongyao Xu, (D) *a Naibo Lin (D) ad \\ and Xiangyang Liu ${ }^{d}$
}

A series of functional polyacetylenes bearing the fluorene moiety with different conjugated lengths and terminal substituents, poly[2-ethynyl-7-(4-nitrostyryl)-9,9-dioctyl-9H-fluorene] (P1), poly[2-ethynyl-7(4-(4-nitrostyryl)styryl)-9,9-dioctyl-9H-fluorene] (P2), poly[2-ethynyl-7-(4-(4-methoxystyryl)styryl)-9,9dioctyl-9H-fluorene] (P3), poly[2-ethynyl-7-(4-(4-methylstyryl)styryl)-9,9-dioctyl-9H-fluorene] (P4), and poly[2-ethynyl-7-(4-(4-methylstyryl)styryl)-9,9-didodecyl-9H-fluorene] (P5), were designed and prepared using $\left[\mathrm{Rh}(\mathrm{nbd}) \mathrm{Cl}_{2}\right.$ as the catalyst. Their structures and properties were characterized and evaluated by FTIR, NMR, UV, FL, GPC and TGA analyses. The optical limiting properties were investigated using 450 fs laser pulses at $780 \mathrm{~nm}$. These results show that the incorporation of styryl/stilbene functionalized polyfluorenylacetylenes has endowed resultant polyacetylenes with novel near-infrared laser protection properties and enhanced thermal stability. The optical limiting mechanism for laser protection was studied. It was found that the optical limiting properties mainly originated from twophoton absorption (TPA) of molecules in the resulting polyacetylenes. Additionally, it was also found that the functionalized polyacetylene with a longer fluorene-based conjugated chromophore and a stronger terminal electron acceptor group exhibits better optical limiting properties because of the larger $\pi$-electron delocalization and dipolar effect.

\section{Introduction}

With the rapid development of new laser technology, laser protection in recent years is believed to be promising for both civilians and the military. ${ }^{1}$ These works have mainly focused on the visible range, where the nonlinear transmission phenomena are mainly dependent on multiphoton absorption (MPA) or reverse saturable absorption (RSA), nonlinear scattering. The materials designed for laser protection involve graphene families, ${ }^{2-4}$ single-walled and multi-walled carbon

${ }^{a}$ College of Material Science and Engineering, State Key Laboratory for Modification of Chemical Fibers and Polymer Materials, Donghua University, Shanghai 201620, China. E-mail: hongyaoxu@163.com

${ }^{b}$ School of Chemistry and Chemical Engineering, The Key Laboratory of Environmentfriendly Polymer Materials of Anhui Province, Anhui University, Hefei 230039, China ${ }^{c}$ College of Chemistry and Bioengineering, Donghua University, Shanghai 201620, China.E-mail:syg@dhu.edu.cn

${ }^{d}$ Department of Physics, Department of Chemistry, National University of Singapore, 2 Science Drive 3, Singapore, 117542, Singapore

$\dagger$ Electronic supplementary information (ESI) available: FTIR, ${ }^{1} \mathrm{H}$ NMR spectra of M1-M3, M5 and P1-P3, P5; ${ }^{13}$ C NMR spectra of M2-M5 and P2-P5; normalized UV-vis absorption and FL emission spectra of M5 (blue solid line) and P5 (red dash dot) in THF solutions; open-aperture Z-scans of P1, P3-P5 at the different pulse energy in THF solution. See DOI: 10.1039/c7ra08499d nanotubes, ${ }^{5-9}$ organic dyes, ${ }^{10}$ inorganic materials, ${ }^{11,12} \pi$-electron conjugated systems such as fullerene $\mathrm{e}^{13,14}$ and porphyrins, phthalocyanines, ${ }^{15-18}$ and organometallic complexes ${ }^{19-21}$ as optical limiting materials for protecting human eyes and sensitive optical sensors from laser damage. Accordingly, to obtain an ideal optical limiting implement, some major factors should be taken into account such as fast response speed, low transmittance at high intensities and high transmittance at low intensities, temporal response and broad spectral characteristics. $^{22}$ For the classical benchmark, porphyrins, phthalocyanines and fullerenes can also provide excellent optical limiting properties. However, their narrow excited-state absorption (ESA) spectral regions and poor optical transparency characteristics restricted practical applications in broadband optical limiting properties. Up to now, a considerable crowd of papers have reported that some materials have broad spectral optical limiting properties under nanosecond photoexcitation. ${ }^{2,23-32}$ Specifically, metal-based materials are premeditated as novel generation optical limiting materials displaying a high transparent characteristic in the visible spectral region. ${ }^{33,34}$ However, to the best of our knowledge, only a few papers reported the laser protection on the near-infrared spectral region has been investigated. The relationship between molecular structure and near-infrared 
laser protection properties has not been understood. ${ }^{\mathbf{1 1 , 3 5 - 3 8}}$ It is well known that substituted polyacetylenes (PAs) are considerable very promising laser protection materials owing to their large third order nonlinear optical susceptibilities, fast time response, good conductivity, ${ }^{39}$ well photophysical stability and processability for optical devices. ${ }^{\mathbf{4 0 - 4 2}}$ In our previous work, many functional polyacetylene materials for laser protection in the visible optical wavelength region were designed. ${ }^{43-52}$ It was found that the incorporation of nonlinear optical chromophore as a pendant group into polyacetylene backbone has endowed polyacetylenes with novel optical limiting properties. The optical limiting properties were mainly influenced by the molecular structure of substituted chromophore group and electron interaction between polyacetylene backbone and substituted chromophore. The interaction generally increases with the decrease of space length between substituted chromophore group and polyacetylene backbone. ${ }^{45}$ The functional polyacetylene containing long conjugated chromophore with $\mathrm{C}=\mathrm{C}$ double bond as $\pi$ electron conjugation bridge exhibited better optical limiting property than that with $\mathrm{C}=\mathrm{N}$ or $\mathrm{N}=\mathrm{N}$ double bond as $\pi$-electron conjugation bridge under the same linear transmittance. ${ }^{47,51}$ Their optical limiting mechanism was attributed to the reverse saturated absorption (RSA) of molecules.

Thus, it was reported that polyacetylenes may be a potential near-infrared laser protection materials. ${ }^{53}$ However, to our knowledge, the polyacetylenes with optical limiting properties for near-infrared optical region have seldom been reported. In particular, the relationship between the structure and nearinfrared optical limiting properties of the molecules, so far, has not been understood. It is known that fluorene is a well near-infrared TPA chromophore. ${ }^{54,55}$ It is expected that the incorporation of fluorene-based chromophores as the pendant groups into polyacetylene could endow the polyacetylenes with novel optical properties. Therefore, in the work, we designed and synthesized a series of novel polyacetylenes that contain fluorene pendant with different conjugation length and terminal substituent such as electron withdrawing or electron donating groups. At the same time, the alkyl chains at nine positions extending away from the conjugated $\pi$-system of fluorene was introduced for improving the solubility of resultant functional polyacetylenes in common organic solvents and provide good compatibility with other matrices. ${ }^{56}$ The relationship between near-infrared optical limiting properties and molecular structure and the optical limiting mechanism were investigated in detail.

\section{Experimental section}

\subsection{Materials}

Unless otherwise noted, all commercial reagents were used as received. 4-Vinylbenzyl chloride, triethyl phosphite, 2-methyl-3butyn-2-ol, $p$-nitrostyrene, palladium(II) acetate, bis(triphenylphosphine) palladium(II) chloride $\left[\mathrm{PdCl}_{2}\left(\mathrm{PPh}_{3}\right)_{2}\right]$, norbornadiene rhodium(I) chloride dimer ([Rh(nbd)Cl $\left.]_{2}\right), \mathrm{WCl}_{6}$, $\mathrm{TaCl}_{5}, \mathrm{MoCl}_{5}$ and tetraphenyltin $\left(\mathrm{Ph}_{4} \mathrm{Sn}\right)$ were all purchased from Aldrich, and used as received without further purification.
2,7-Dibromofluorene was obtained from TEIKO International LTD. 1-Bromooctane, 1-bromododecane, tetrabutyl ammonium bromide, 4-methoxybenzaldehyde, 4-methylbenzaldehyde, 4nitrobenzaldehyde, $\mathrm{NaH}, \mathrm{NaOH}, \mathrm{KOH}, \mathrm{MgSO}_{4}$, triphenylphosphine, tetrahydrofuran (THF), dimethylsulfoxide (DMSO), dimethylformamide (DMF), triethylamine $\left(\mathrm{Et}_{3} \mathrm{~N}\right)$, dioxane, dichloromethane, petroleum ether and ethyl acetate were all purchased from Shanghai Chemical Reagent Company. Dioxane and THF were distilled from sodium benzophenone ketyl immediately before use. DMF was dried over anhydrous $\mathrm{MgSO}_{4}$ and freshly distilled prior to use. $\mathrm{Et}_{3} \mathrm{~N}$ was distilled from $\mathrm{KOH}$ before use. Technical grade methanol was used to precipitate the polymers.

\subsection{Instrumentation}

FTIR spectra of KBr disks were recorded with a Thermo Nicolet 5700 spectrometer at room temperature, 32 scans were collected at a resolution of $1 \mathrm{~cm}^{-1} \cdot{ }^{1} \mathrm{H}$ NMR (400 MHz) and ${ }^{13} \mathrm{C}$ NMR (100 $\mathrm{MHz}$ ) were recorded on a Bruker DMX-400 spectrometer utilizing tetramethylsilane (TMS, $0.00 \mathrm{ppm}$ ) as the internal standard in chloroform-d $\left(\mathrm{CDCl}_{3}\right)$ at room temperature. Elementary analyses were conducted on Vario EL-III elementary analysis apparatus. UV-vis spectra were recorded on a Shimadzu UV-265 spectrometer using a $1 \mathrm{~cm}$ square quartz cell. Fluorescence spectra were obtained on a Shimadzu RF-5301PC spectrofluorimeter equipped with a $450 \mathrm{~W}$ Xe lamp and a timecorrelated single-photon counting card. Weight-average $\left(M_{\mathrm{w}}\right)$ and number-average $\left(M_{\mathrm{n}}\right)$ molecular weights, and polydispersity index (PDI, $M_{\mathrm{w}} / M_{\mathrm{n}}$ ) were determined by gel permeation chromatograph (GPC) using a Waters 510 HPLC equipped with a Rheodyne 7725i injector with a stand kit, a set of styragel columns (HT3, HT4, and HT6; molecular weight range $10^{2}-10^{7}$ ), a column temperature controller, a Waters 486 wavelength tunable UV-vis detector, a Waters 410 differential refractometer, and a system DMM/scanner possessing an 8-channel scanner option. All polymer solutions were prepared in THF (ca. $2 \mathrm{mg} \mathrm{mL} \mathrm{mL}^{-1}$ ) and filtered through $0.45 \mu \mathrm{m}$ PTFE syringe-type filters before injected into the GPC system. THF was used as the eluent at a flow rate of $1.0 \mathrm{~mL} \mathrm{~min}^{-1}$. The column temperature was maintained at $30{ }^{\circ} \mathrm{C}$ and the working wavelength of the UV detector was set at $254 \mathrm{~nm}$. A set of mono-disperse polystyrene standards (waters) was used for calibration purposes. Differential scanning calorimetry (DSC) was performed on a TA Instruments Q2000 equipped with a liquid nitrogen cooling accessory (LNCA) unit under a continuous nitrogen purge $\left(50 \mathrm{~mL} \min ^{-1}\right)$. The scan rate was $10{ }^{\circ} \mathrm{C} \min ^{-1}$ within the temperature range $30-250{ }^{\circ} \mathrm{C}$. Samples $(4-6 \mathrm{mg}$ ) were weighed and sealed in aluminum pans. Thermogravimetric analysis (TGA) was carried out using a NETZSCH Instruments $449 \mathrm{~F} 3$ thermogravimetric analyzer with a heating rate of $20{ }^{\circ} \mathrm{C} \mathrm{min}-1$ from 20 to $800{ }^{\circ} \mathrm{C}$ under a continuous nitrogen purge $\left(100 \mathrm{~mL} \mathrm{~min}^{-1}\right)$. Samples (15-25 mg) were loaded in alumina pans. The thermal degradation temperature $\left(T_{\mathrm{d}}\right)$ was defined as the temperature of $5 \%$ mass loss.

The molecular two-photon absorption (TPA) cross sections were measured by a Z-scan technique. The laser pulses were 
produced by a mode-locked Ti:sapphire laser (Quantronix, IMRA), which seeded a Ti:sapphire regenerative amplifier, and focused onto a $1 \mathrm{~mm}$-thick quartz cuvette containing the solutions of these molecules with the minimum beam waist of $25 \pm$ $5 \mu \mathrm{m}$. The incident and transmitted laser pulse energy were monitored by moving the cuvette along the propagation direction of the laser pulses. The maximum on-axis irradiance of the laser pulses was $\sim 120 \mathrm{GW} \mathrm{cm}^{-2}$ at the focus point. The Z-scan experimental system was calibrated using a piece of cadmium sulfide (CdS) bulk crystal as a reference because it possesses large TPA at the wavelength of $780 \mathrm{~nm}$ and has been well investigated in our laboratory. The TPA coefficient of CdS was determined to be $6.4 \pm 0.6 \mathrm{~cm} \mathrm{GW}^{-1}$, which was in good agreement with theoretical values within the experiment uncertainty. The investigation of the optical limiting properties of the samples was carried out by using the same laser system as in the Z-scan experiment. The experimental arrangement was similar with that in the literature. ${ }^{57}$ The samples were contained in quartz cells with a thickness of $1 \mathrm{~mm}$ and positioned at the focal point. Two-photon fluorescence (TPF) measurements of the polymers in solid form were carried out using the same laser system described above. The details of the two-photon technique experimental setup are described elsewhere. ${ }^{58}$

\subsection{Monomer synthesis (cf. Scheme 1)}

4-Ethenyl-4' -nitro-1,2-stilbene (1a). $3.9 \mathrm{~mL}$ 4-vinylbenzyl chloride $(25 \mathrm{mmol})$ and $5.5 \mathrm{~mL}$ triethyl-phosphite $(30 \mathrm{mmol})$ were heated at $110{ }^{\circ} \mathrm{C}$ for $6 \mathrm{~h}$ in a solvent-free system. Excess triethylphosphite was removed by vacuum distillation, and the resultant phosphonate was obtained as the thick oil, which was used for the Wittig-Horner reaction without further purification. After the crude phosphonate in $50 \mathrm{~mL}$ of dry THF was cooled to $0{ }^{\circ} \mathrm{C}, 1.2 \mathrm{~g} \mathrm{NaH}(50 \mathrm{mmol})$ were added slowly and reacted for $30 \mathrm{~min} .3 .78 \mathrm{~g}$ 4-nitrobenzaldehyde $(25 \mathrm{mmol})$ in $10 \mathrm{~mL}$ THF was then added dropwise, and the contents were stirred under nitrogen for $12 \mathrm{~h}$. The mixture was added slowly to $150 \mathrm{~mL}$ of ice-chilled water, and the red brown solid was filtered and rinsed twice with water and ethanol. The crude product was recrystallized from THF/ethanol $(2: 1 \mathrm{v} / \mathrm{v})$ to provide red brown crystal powder. Yield: 53\%. IR (KBr), $\nu\left(\mathrm{cm}^{-1}\right): 3021,997,927$, 972 (=C-H), 1629 (C=C), 1589, $850(\mathrm{Ar}), 1514,1338\left(\mathrm{NO}_{2}\right) \cdot{ }^{1} \mathrm{H}$ NMR (400 MHz, $\mathrm{CDCl}_{3}$ ), $\delta$ (TMS, ppm): 8.24 (d, $J=8.8 \mathrm{~Hz}, 2 \mathrm{H}$, Ar-H ortho to $\left.\mathrm{NO}_{2}\right), 7.65\left(\mathrm{~d}, J=8.8 \mathrm{~Hz}, 2 \mathrm{H}\right.$, Ar-H meta to $\mathrm{NO}_{2}$ ), 7.54 (d, $J=8.4 \mathrm{~Hz}, 2 \mathrm{H}$, Ar-H meta to vinyl), 7.46 (d, $J=8.4 \mathrm{~Hz}$, $2 \mathrm{H}$, Ar-H ortho to vinyl), 7.30-7.14 (q, $J=18.7 \mathrm{~Hz}, 2 \mathrm{H},-\mathrm{CH}=$ $\mathrm{CH}-$ ), 6.79-6.72 (q, $\left.J=9.5 \mathrm{~Hz}, 1 \mathrm{H},-\mathrm{CH}=\mathrm{CH}_{2}\right), 5.82(\mathrm{~d}, J=$ $\left.17.6 \mathrm{~Hz}, 1 \mathrm{H},-\mathrm{CH}=\mathrm{CH}_{2}\right), 5.33\left(\mathrm{~d}, J=11.2 \mathrm{~Hz}, 1 \mathrm{H},-\mathrm{CH}=\mathrm{CH}_{2}\right)$.

4-Ethenyl-4'-methoxy-1,2-stilbene (1b). The synthesis process is similar to compound $\mathbf{1 a}$ by using 4-methoxybenzaldehyde. The product was gray crystal. Yield: 39\%. IR $(\mathrm{KBr}), \nu\left(\mathrm{cm}^{-1}\right): 3001,970,992,905(=\mathrm{C}-\mathrm{H}), 2954,2834\left(\mathrm{CH}_{3}\right)$, $1624(\mathrm{C}=\mathrm{C}), 1601,1514,1462,840(\mathrm{Ar}), 1254,1036(\mathrm{C}-\mathrm{O}-\mathrm{C}) .{ }^{1} \mathrm{H}$ NMR (400 MHz, $\mathrm{CDCl}_{3}$ ), $\delta$ (TMS, ppm): $7.48(\mathrm{~d}, J=8.8 \mathrm{~Hz}, 4 \mathrm{H}$, $\left.\mathrm{Ar}-\mathrm{H}-\mathrm{CH}=\mathrm{CH}_{2}\right) 7.42\left(\mathrm{~d}, J=8.4 \mathrm{~Hz}, 2 \mathrm{H}\right.$, Ar-H meta to $\left.\mathrm{OCH}_{3}\right)$, 7.11-6.96 (q, $J=13.6 \mathrm{~Hz}, 2 \mathrm{H}$, Ar-H ortho to $\left.\mathrm{OCH}_{3}\right), 6.92(\mathrm{~d}, J=$ $8.8 \mathrm{~Hz}, 2 \mathrm{H},-\mathrm{CH}=\mathrm{CH}-), 6.74\left(\mathrm{q}, J=17.6 \mathrm{~Hz}, 1 \mathrm{H},-\mathrm{CH}=\mathrm{CH}_{2}\right)$, $5.77\left(\mathrm{~d}, J=17.6 \mathrm{~Hz}, 1 \mathrm{H},-\mathrm{CH}=\mathrm{CH}_{2}\right), 5.26(\mathrm{~d}, J=12.0 \mathrm{~Hz}, 1 \mathrm{H}$, $\left.-\mathrm{CH}=\mathrm{CH}_{2}\right), 3.86\left(\mathrm{~s}, 3 \mathrm{H}, \mathrm{Ar}-\mathrm{OCH}_{3}\right)$.

4-Ethenyl-4'-methyl-1,2-stilbene (1c). The synthesis process is similar to compound 1a by using 4-methylbenzaldehyde. The product was white crystal. Yield: $44 \%$. IR $(\mathrm{KBr}), \nu\left(\mathrm{cm}^{-1}\right): 3021$, 970, 992, 898 (=C-H), 2915, $2855\left(\mathrm{CH}_{3}\right), 1624$ (C=C), 1513, 835 (Ar). ${ }^{1} \mathrm{H}$ NMR (400 MHz, $\mathrm{CDCl}_{3}$ ), $\delta$ (TMS, ppm): $7.48(\mathrm{~d}, J=$ $8.4 \mathrm{~Hz}, 2 \mathrm{H}$, Ar-H meta to $\left.\mathrm{CH}_{3}\right), 7.43\left(\mathrm{t}, J=13.6 \mathrm{~Hz}, 4 \mathrm{H}, \mathrm{Ar}-\mathrm{H}^{-}\right.$ $\left.\mathrm{CH}=\mathrm{CH}_{2}\right) 7.19\left(\mathrm{~d}, J=8.0 \mathrm{~Hz}, 2 \mathrm{H}, \mathrm{Ar}-\mathrm{H}\right.$ ortho to $\left.\mathrm{CH}_{3}\right), 7.08(\mathrm{~d}, J=$ $6.0 \mathrm{~Hz}, 2 \mathrm{H},-\mathrm{CH}=\mathrm{CH}-), 6.74\left(\mathrm{q}, J=18.2 \mathrm{~Hz}, 1 \mathrm{H},-\mathrm{CH}=\mathrm{CH}_{2}\right)$, $5.77\left(\mathrm{~d}, J=17.6 \mathrm{~Hz}, 1 \mathrm{H},-\mathrm{CH}=\mathrm{CH}_{2}\right), 5.26(\mathrm{~d}, J=11.2 \mathrm{~Hz}, 1 \mathrm{H}$, $\left.-\mathrm{CH}=\mathrm{CH}_{2}\right), 2.39\left(\mathrm{~s}, 3 \mathrm{H}, \mathrm{Ar}-\mathrm{CH}_{3}\right)$.

2,7-Dibromo-9,9-dioctylfluorene (2a). 1-Bromooctane (5.60 g, $29.0 \mathrm{mmol}$ ) was added by using a syringe to a mixture of $2,7-$ dibromofluorene $(3.88 \mathrm{~g}, 12.0 \mathrm{mmol})$, tetrabutyl ammonium bromide $(0.0225 \mathrm{~g}, 0.0975 \mathrm{mmol})$ and $3.75 \mathrm{~mL}$ of $50 \%$ aqueous $\mathrm{NaOH}$ in DMSO $(50 \mathrm{~mL})$. The reaction mixture was stirred at room temperature for $3 \mathrm{~h}$. The mixture was poured into $\mathrm{H}_{2} \mathrm{O}$ $(500 \mathrm{~mL})$, and then was extracted three times with dichloromethane. The combined organic layers were dried over anhydrous $\mathrm{MgSO}_{4}$ and decolor by active carbon. The solvent was removed under reduced pressure. The crude product was purified by recrystallization by hexane as the solvent to yield a colorless crystal. Yield: $89 \%$. IR (KBr), $\nu\left(\mathrm{cm}^{-1}\right): 2955,2920$, $2851\left(\mathrm{CH}_{2}, \mathrm{CH}_{3}\right) ; 1467,1448,1416,812$ (Ar). ${ }^{1} \mathrm{H}$ NMR $(400 \mathrm{MHz}$, $\mathrm{CDCl}_{3}$ ), $\delta$ (TMS, ppm): 7.51 (d, 2H, $\left.J=7.7 \mathrm{~Hz}\right), 7.45(\mathrm{~m}, 4 \mathrm{H}), 1.90$ $\left(\mathrm{m}, 4 \mathrm{H},-\mathrm{CH}_{2}\left(\mathrm{CH}_{2}\right)_{6} \mathrm{CH}_{3}\right), 1.25-0.86\left(\mathrm{~m}, 20 \mathrm{H},-\mathrm{CH}_{2}\left(\mathrm{CH}_{2}\right)_{5} \mathrm{C}_{2} \mathrm{H}_{5}\right)$, $0.83\left(\mathrm{t}, 6 \mathrm{H}, J=7.2 \mathrm{~Hz},-\left(\mathrm{CH}_{2}\right)_{7} \mathrm{CH}_{3}\right), 0.58(\mathrm{~s}, 4 \mathrm{H}$, $\left.-\left(\mathrm{CH}_{2}\right)_{6} \mathrm{CH}_{2} \mathrm{CH}_{3}\right)$.

2,7-Dibromo-9,9-1-didodecylfluorene (2b). The synthesis process is similar to compound 2 a by using 1-bromododecane. Yield: 78\%. IR (KBr), $\nu\left(\mathrm{cm}^{-1}\right): 2935,2923,2850\left(\mathrm{CH}_{2}, \mathrm{CH}_{3}\right)$; 1464, 1449, 1416, 808 (Ar). ${ }^{1} \mathrm{H}$ NMR (400 MHz, $\mathrm{CDCl}_{3}$ ), $\delta$ (TMS, ppm): $7.51(\mathrm{~d}, 2 \mathrm{H}, J=7.7 \mathrm{~Hz}), 7.45(\mathrm{~m}, 4 \mathrm{H}), 1.90(\mathrm{~m}, 4 \mathrm{H}$, $\left.-\mathrm{CH}_{2}\left(\mathrm{CH}_{2}\right)_{10} \mathrm{CH}_{3}\right), 1.30-1.00\left(\mathrm{~m}, 36 \mathrm{H},-\mathrm{CH}_{2}\left(\mathrm{CH}_{2}\right)_{9} \mathrm{C}_{2} \mathrm{H}_{5}\right), 0.87(\mathrm{t}$, $\left.J=7.2 \mathrm{~Hz}, 6 \mathrm{H},-\left(\mathrm{CH}_{2}\right)_{11} \mathrm{CH}_{3}\right), 0.59\left(\mathrm{~s}, 4 \mathrm{H},-\left(\mathrm{CH}_{2}\right)_{10} \mathrm{CH}_{2} \mathrm{CH}_{3}\right)$.

2-Bromo-7-(4-nitrostyryl)-9,9-dioctyl-9H-fluorene (3). 2,7Dibromo-9,9-dioctylfluorene (1.096 g, $2.0 \mathrm{mmol}), \mathrm{Pd}(\mathrm{OAc})_{2}$ (4.48 mg, $2.0 \times 10^{-2} \mathrm{mmol}$ ), triphenylphosphine (10.50 mg, 4.0 $\left.\times 10^{-2} \mathrm{mmol}\right)$ and $p$-nitrostyrene $(0.3 \mathrm{~mL}, 2.0 \mathrm{mmol})$ were dissolved in $20 \mathrm{~mL} \mathrm{DMF}$ and $4 \mathrm{~mL} \mathrm{Et}_{3} \mathrm{~N}$. The resultant mixture was combined in a screw cap vial and heated for $48 \mathrm{~h}$ at $110{ }^{\circ} \mathrm{C}$ under a nitrogen atmosphere. After cooling to room temperature, the precipitate was filtered and the filtrate was concentrated. The mixture was extracted with $\mathrm{CH}_{2} \mathrm{Cl}_{2}$ and dried over $\mathrm{MgSO}_{4}$. The crude product was purified by silica gel column chromatography using petroleum ether/dichloromethane $(6: 1$ $\mathrm{v} / \mathrm{v}$ ) as the eluent. Product was obtained as a yellow solid. Yield: $62 \%$. IR (KBr), $\nu\left(\mathrm{cm}^{-1}\right): 2975,2922\left(\mathrm{CH}_{2}, \mathrm{CH}_{3}\right), 1604,1512,856$ (Ar), 1517, $1346\left(\mathrm{NO}_{2}\right), 1062$ (Ar-Br). ${ }^{1} \mathrm{H} \mathrm{NMR}\left(400 \mathrm{MHz}, \mathrm{CDCl}_{3}\right.$ ), $\delta$ (TMS, ppm): $8.23(\mathrm{~d}, J=8.8 \mathrm{~Hz}, 2 \mathrm{H}), 7.70-7.42(\mathrm{~m}, 8 \mathrm{H}), 7.35(\mathrm{~d}$, $J=16.0 \mathrm{~Hz}, 1 \mathrm{H}), 7.21(\mathrm{~d}, J=16.0 \mathrm{~Hz}, 1 \mathrm{H}), 1.91(\mathrm{~m}, 4 \mathrm{H}$, $\left.-\mathrm{CH}_{2}\left(\mathrm{CH}_{2}\right)_{6} \mathrm{CH}_{3}\right), 1.25-0.86\left(\mathrm{~m}, 20 \mathrm{H},-\mathrm{CH}_{2}\left(\mathrm{CH}_{2}\right)_{5} \mathrm{C}_{2} \mathrm{H}_{5}\right), 0.84(\mathrm{t}$, $\left.J=7.2 \mathrm{~Hz}, 6 \mathrm{H},-\left(\mathrm{CH}_{2}\right)_{7} \mathrm{CH}_{3}\right), 0.58\left(\mathrm{~m}, 4 \mathrm{H},-\left(\mathrm{CH}_{2}\right)_{6} \mathrm{CH}_{2} \mathrm{CH}_{3}\right)$.

2-Bromo-7-(4-(4-nitrostyryl)styryl)-9,9-dioctyl-9H-fluorene (4a). The synthesis process is similar to compound 3 by using 4-ethenyl-4'-nitro-1,2-stilbene (1a). The crude product was 
purified by silica gel column chromatography using petroleum ether/dichloromethane $(5: 1 \mathrm{v} / \mathrm{v})$ as the eluent. Product was obtained as a yellowish-red solid. Yield: $79 \%$. IR $(\mathrm{KBr}), \nu\left(\mathrm{cm}^{-1}\right)$ : 3023, 962 (=C-H), 2955, 2924, $2852\left(\mathrm{CH}_{2}, \mathrm{CH}_{3}\right), 1588,1460,816$ (Ar), 1514, $1338\left(\mathrm{NO}_{2}\right), 1063$ (Ar-Br). ${ }^{1} \mathrm{H}$ NMR $\left(400 \mathrm{MHz}, \mathrm{CDCl}_{3}\right)$, $\delta$ (TMS, ppm): 8.25 (d, $J=8.4 \mathrm{~Hz}, 2 \mathrm{H}) 7.68-7.46(\mathrm{~m}, 12 \mathrm{H}), 7.33-$ 7.17 (q, 4H), $1.98\left(\mathrm{~m}, 4 \mathrm{H},-\mathrm{CH}_{2}\left(\mathrm{CH}_{2}\right)_{6} \mathrm{CH}_{3}\right), 1.25-1.09(\mathrm{~m}, 20 \mathrm{H}$, $\left.-\mathrm{CH}_{2}\left(\mathrm{CH}_{2}\right)_{5} \mathrm{C}_{2} \mathrm{H}_{5}\right), 0.84\left(\mathrm{t}, J=6.8 \mathrm{~Hz}, 6 \mathrm{H},-\left(\mathrm{CH}_{2}\right)_{7} \mathrm{CH}_{3}\right), 0.65(\mathrm{~m}$, $\left.4 \mathrm{H},-\left(\mathrm{CH}_{2}\right)_{6} \mathrm{CH}_{2} \mathrm{CH}_{3}\right)$.

2-Bromo-7-(4-(4-methoxystyryl)styryl)-9,9-dioctyl-9H-fluorene (4b). The synthesis process is similar to compound 3 by using 4 ethenyl-4'-methoxy-1,2-stilbene (1b). The crude product was purified by silica gel column chromatography using petroleum ether/dichloromethane $(10: 1 \mathrm{v} / \mathrm{v})$ as the eluent. Product was obtained as a yellow solid. Yield: $68 \%$. IR $(\mathrm{KBr}), \nu\left(\mathrm{cm}^{-1}\right): 3022$, $963(=\mathrm{C}-\mathrm{H}), 2953,2926,2852\left(\mathrm{CH}_{2}, \mathrm{CH}_{3}\right), 1603,1512,1458,825$ (Ar), 1250, 1033 (C-O-C), 1061 (Ar-Br). ${ }^{1} \mathrm{H}$ NMR $(400 \mathrm{MHz}$, $\mathrm{CDCl}_{3}$ ), $\delta$ (TMS, ppm): 7.66 (d, $\left.J=7.6 \mathrm{~Hz}, 1 \mathrm{H}\right) 7.57-7.46(\mathrm{~m}$, $11 \mathrm{H}), 7.21-6.92(\mathrm{~m}, 6 \mathrm{H}), 3.87\left(\mathrm{~s}, 3 \mathrm{H}, \mathrm{Ar}^{-} \mathrm{OCH}_{3}\right), 1.98(\mathrm{~m}, 4 \mathrm{H}$, $\left.-\mathrm{CH}_{2}\left(\mathrm{CH}_{2}\right)_{6} \mathrm{CH}_{3}\right), 1.23-1.08\left(\mathrm{~m}, 20 \mathrm{H},-\mathrm{CH}_{2}\left(\mathrm{CH}_{2}\right)_{5} \mathrm{C}_{2} \mathrm{H}_{5}\right), 0.83(\mathrm{t}$, $\left.J=7.2 \mathrm{~Hz}, 6 \mathrm{H},-\left(\mathrm{CH}_{2}\right)_{7} \mathrm{CH}_{3}\right), 0.65\left(\mathrm{~m}, 4 \mathrm{H},-\left(\mathrm{CH}_{2}\right)_{6} \mathrm{CH}_{2} \mathrm{CH}_{3}\right)$.

2-Bromo-7-(4-(4-methylstyryl)styryl)-9,9-dioctyl-9H-fluorene (4c). The synthesis process is similar to compound 3 by using 4 ethenyl-4'-methyl-1,2-stilbene (1c). The crude product was purified by silica gel column chromatography using petroleum ether/dichloromethane $(20: 1 \mathrm{v} / \mathrm{v})$ as the eluent. Product was obtained as a yellow solid. Yield: $65 \%$. IR $(\mathrm{KBr}), \nu\left(\mathrm{cm}^{-1}\right): 3023$, $962(=\mathrm{C}-\mathrm{H}), 2953,2926,2852\left(\mathrm{CH}_{2}, \mathrm{CH}_{3}\right), 1514,1458,820(\mathrm{Ar})$, 1062 (Ar-Br). ${ }^{1} \mathrm{H}$ NMR (400 MHz, $\mathrm{CDCl}_{3}$ ), $\delta$ (TMS, ppm): 7.66 (d, $J$ $=7.6 \mathrm{~Hz}, 1 \mathrm{H}) 7.57-7.44(\mathrm{~m}, 11 \mathrm{H}), 7.20(\mathrm{~m}, 4 \mathrm{H}), 7.11(\mathrm{~d}, 2 \mathrm{H}), 2.39$ (s, 3H, Ar-CH $\left.H_{3}\right), 1.98\left(\mathrm{~m}, 4 \mathrm{H},-\mathrm{CH}_{2}\left(\mathrm{CH}_{2}\right)_{6} \mathrm{CH}_{3}\right), 1.25-1.09$ (m, $\left.20 \mathrm{H},-\mathrm{CH}_{2}\left(\mathrm{CH}_{2}\right)_{5} \mathrm{C}_{2} \mathrm{H}_{5}\right), 0.84\left(\mathrm{t}, J=6.8 \mathrm{~Hz}, 6 \mathrm{H},-\left(\mathrm{CH}_{2}\right)_{7} \mathrm{CH}_{3}\right), 0.65$ (m, $\left.4 \mathrm{H},-\left(\mathrm{CH}_{2}\right)_{6} \mathrm{CH}_{2} \mathrm{CH}_{3}\right)$.

2-Bromo-7-(4-(4-methylstyryl)styryl)-9,9-didodecyl-9H-fluorene (4d). The synthesis process is similar to compound 3 by using 4-ethenyl-4'-methyl-1,2-stilbene (1c) and 2,7-dibromo-9,9didodecylfluorene (2b). The crude product was purified by silica gel column chromatography using petroleum ether/ dichloromethane $(25: 1 \mathrm{v} / \mathrm{v})$ as the eluent. Product was obtained as a yellow solid. Yield: 54\%. IR (KBr), $\nu\left(\mathrm{cm}^{-1}\right): 3023,965$ (=C-H); 2926, $2852\left(\mathrm{CH}_{2}, \mathrm{CH}_{3}\right)$; 1514, 1457, 821 (Ar), 1061 (ArBr). ${ }^{1} \mathrm{H}$ NMR $\left(400 \mathrm{MHz}, \mathrm{CDCl}_{3}\right.$ ), $\delta$ (TMS, ppm): 7.66 (d, $J=$ $8.0 \mathrm{~Hz}, 1 \mathrm{H})$ 7.57-7.44 (m, 11H), 7.26-7.07 (m, 6H), $2.39(\mathrm{~s}, 3 \mathrm{H}$, $\left.\mathrm{Ar}-\mathrm{CH}_{3}\right), 1.98\left(\mathrm{~m}, 4 \mathrm{H},-\mathrm{CH}_{2}\left(\mathrm{CH}_{2}\right)_{10} \mathrm{CH}_{3}\right), 1.23-1.08(\mathrm{~m}, 36 \mathrm{H}$, $\left.-\mathrm{CH}_{2}\left(\mathrm{CH}_{2}\right)_{9} \mathrm{C}_{2} \mathrm{H}_{5}\right), 0.88\left(\mathrm{t}, J=7.2 \mathrm{~Hz}, 6 \mathrm{H},-\left(\mathrm{CH}_{2}\right)_{11} \mathrm{CH}_{3}\right), 0.66(\mathrm{~m}$, $\left.4 \mathrm{H},-\left(\mathrm{CH}_{2}\right)_{10} \mathrm{CH}_{2} \mathrm{CH}_{3}\right)$.

2-Ethynyl-7-(4-nitrostyryl)-9,9-dioctyl-9H-fluorene (M1). 2Methyl-3-butyn-2-ol $(0.8 \mathrm{~mL}, 8.0 \mathrm{mmol})$ was added to a solution of $\mathrm{PdCl}_{2}\left(\mathrm{PPh}_{3}\right)_{2}\left(28.00 \mathrm{mg}, 4.0 \times 10^{-2} \mathrm{mmol}\right)$, $\mathrm{CuI}(2.00 \mathrm{mg}, 1.0$ $\left.\times 10^{-2} \mathrm{mmol}\right)$, and $3(0.38 \mathrm{~g}, 0.5 \mathrm{mmol})$ in $20 \mathrm{~mL}$ of triethylamine under a nitrogen atmosphere. The mixture was reacted at $80{ }^{\circ} \mathrm{C}$ for $48 \mathrm{~h}$. After cooling to room temperature, the precipitate was filtered and the filtrate was concentrated. The crude product was purified by aluminum oxide column using ethyl acetate/petroleum ether mixture $(1: 6 \mathrm{v} / \mathrm{v})$ as eluent. The resulting solid 2-(2-methyl-3-yn-2-ol)-7-(4-nitrostyryl)-9,9-dioctyl-9H-fluorene, was a yellow powder in $60 \%$ yield. Sodium hydroxide (144 mg, $3.6 \mathrm{mmol}$ ) was added with stirring to a solution of 2-(2-methyl-3-yn-2-ol)-7-(4-nitrostyryl)-9,9-dioctyl$9 H$-fluorene $(0.39 \mathrm{~g}, 0.5 \mathrm{mmol})$ in dry dioxane $(20.0 \mathrm{~mL})$ under nitrogen. The reaction mixture was then heated at $80{ }^{\circ} \mathrm{C}$ under reflux for $4 \mathrm{~h}$. After cooling to room temperature and the removal of the solvent with a rotary evaporator, the raw product was extracted with dichloromethane three times, dried over $\mathrm{MgSO}_{4}$ and recrystallized from dichloromethane/ethanol mixture $(2: 1 \mathrm{v} / \mathrm{v})$. Product was obtained as a yellow powder. Yield: $85 \%$. Mp: $120.0^{\circ} \mathrm{C}$. IR (KBr), $\nu\left(\mathrm{cm}^{-1}\right): 3310(\equiv \mathrm{C}-\mathrm{H}), 2974$, $2922\left(\mathrm{CH}_{2}, \mathrm{CH}_{3}\right), 2103(\mathrm{C} \equiv \mathrm{C}), 1602,1512,850(\mathrm{Ar}), 1516,1343$ $\left(\mathrm{NO}_{2}\right) .{ }^{1} \mathrm{H}$ NMR $\left(400 \mathrm{MHz}, \mathrm{CDCl}_{3}\right), \delta$ (TMS, ppm): 8.26 (d, $J=$ $8.0 \mathrm{~Hz}, 2 \mathrm{H}), 7.61-7.53(\mathrm{~m}, 4 \mathrm{H}), 7.51-7.42(\mathrm{~m}, 4 \mathrm{H}), 7.28(\mathrm{~d}, J=$ $16.0 \mathrm{~Hz}, 1 \mathrm{H}), 7.12(\mathrm{~d}, J=16.0 \mathrm{~Hz}, 1 \mathrm{H}), 3.18(\mathrm{~s}, 1 \mathrm{H},-\mathrm{C} \equiv \mathrm{CH}), 1.96$ $\left(\mathrm{m}, 4 \mathrm{H},-\mathrm{CH}_{2}\left(\mathrm{CH}_{2}\right)_{6} \mathrm{CH}_{3}\right), 1.04\left(\mathrm{~m}, 20 \mathrm{H},-\mathrm{CH}_{2}\left(\mathrm{CH}_{2}\right)_{5} \mathrm{C}_{2} \mathrm{H}_{5}\right), 0.61$ $\left(\mathrm{t}, J=7.2 \mathrm{~Hz}, 6 \mathrm{H},-\left(\mathrm{CH}_{2}\right)_{7} \mathrm{CH}_{3}\right), 0.56\left(\mathrm{~m}, 4 \mathrm{H},-\left(\mathrm{CH}_{2}\right)_{6} \mathrm{CH}_{2} \mathrm{CH}_{3}\right)$. Anal. calcd for $\mathrm{C}_{39} \mathrm{H}_{37} \mathrm{NO}_{2}$ : C 84.90, H 6.75, N 2.53. Found: C 84.89, H: 6.77, N 2.51.

2-Ethynyl-7-(4-(4-nitrostyryl)styryl)-9,9-dioctyl-9H-fluorene (M2). This compound was prepared as the similar approach of M1, using 4a instead of 3, as the starting material. Product was recrystallized from dichloromethane/ethanol mixture $(2: 1 \mathrm{v} / \mathrm{v})$, and obtained as an orange-red powder. Yield: $65 \%$. Mp: $136.1{ }^{\circ} \mathrm{C}$. IR (KBr), $\nu\left(\mathrm{cm}^{-1}\right): 3318(\equiv \mathrm{C}-\mathrm{H}), 3022,961(=\mathrm{C}-\mathrm{H}), 2923,2851\left(\mathrm{CH}_{2}, \mathrm{CH}_{3}\right)$, $2104(\mathrm{C} \equiv \mathrm{C}), 1584,1466,821$ (Ar), 1511, $1339\left(\mathrm{NO}_{2}\right) .{ }^{1} \mathrm{H}$ NMR (400 $\left.\mathrm{MHz}, \mathrm{CDCl}_{3}\right), \delta(\mathrm{TMS}, \mathrm{ppm}): 8.25(\mathrm{~d}, J=8.4 \mathrm{~Hz}, 2 \mathrm{H}), 7.70-7.50(\mathrm{~m}$, $12 \mathrm{H}), 7.32-7.17(\mathrm{q}, 4 \mathrm{H}), 3.18(\mathrm{~s}, 1 \mathrm{H},-\mathrm{C} \equiv \mathrm{CH}), 2.00(\mathrm{~m}, 4 \mathrm{H}$, $\left.-\mathrm{CH}_{2}\left(\mathrm{CH}_{2}\right)_{6} \mathrm{CH}_{3}\right), 1.24-1.08\left(\mathrm{~m}, 20 \mathrm{H},-\mathrm{CH}_{2}\left(\mathrm{CH}_{2}\right)_{5} \mathrm{C}_{2} \mathrm{H}_{5}\right), 0.84(\mathrm{t}, J=$ $\left.7.2 \mathrm{~Hz}, 6 \mathrm{H},-\left(\mathrm{CH}_{2}\right)_{7} \mathrm{CH}_{3}\right), 0.65\left(\mathrm{~m}, 4 \mathrm{H},-\left(\mathrm{CH}_{2}\right)_{6} \mathrm{CH}_{2} \mathrm{CH}_{3}\right) .{ }^{13} \mathrm{C} \mathrm{NMR}$ (75 MHz, $\mathrm{CDCl}_{3}$ ), $\delta$ (ppm): 151.7, 151.0, 146.8, 143.9, 141.5, 140.4, 138.1, 136.7, 135.4, 132.9, 131.2, 129.9, 127.6, 127.5, 127.0, 126.8, $126.5,126.0,125.8,124.2,120.9,120.4,120.3,119.6$ ( $\mathrm{Ar}$ and $-\mathrm{CH}=$ $\mathrm{CH}-), 84.7(-\mathrm{C} \equiv \mathrm{CH}), 77.2(-\mathrm{C} \equiv \mathrm{CH}), 55.1,40.4,31.8,30.0,29.2$, 23.7, 22.6, $14.1\left(-\left(\mathrm{CH}_{2}\right)_{7} \mathrm{CH}_{3}\right)$. Anal. calcd for $\mathrm{C}_{47} \mathrm{H}_{53} \mathrm{NO}_{2}$ : C 85.02, H 8.05, N 2.11. Found: C 85.09, H: 8.01, N 2.07.

2-Ethynyl-7-(4-(4-methoxystyryl)styryl)-9,9-dioctyl-9H-fluorene (M3). This compound was prepared as the similar approach of M1, using $\mathbf{4 b}$ instead of $\mathbf{3}$, as the starting material. Product was recrystallized from dichloromethane/ethanol mixture $(1: 1 \mathrm{v} / \mathrm{v})$, and obtained as a yellow powder. Yield: $73 \%$. Mp: $135.8^{\circ} \mathrm{C}$. IR $(\mathrm{KBr}), \nu\left(\mathrm{cm}^{-1}\right): 3310(\equiv \mathrm{C}-\mathrm{H}), 3021,968(=\mathrm{C}-\mathrm{H}), 2926,2852$ $\left(\mathrm{CH}_{2}, \mathrm{CH}_{3}\right), 2104(\mathrm{C} \equiv \mathrm{C}), 1605,1512,1462,830(\mathrm{Ar}), 1255,1038$ (C-O-C). ${ }^{1} \mathrm{H}$ NMR (400 MHz, $\mathrm{CDCl}_{3}$ ), $\delta$ (TMS, ppm): 7.70-7.64 (q, $J=7.8 \mathrm{~Hz}, 2 \mathrm{H}) 7.57-7.49(\mathrm{~m}, 10 \mathrm{H}), 7.26-6.92(\mathrm{~m}, 6 \mathrm{H}), 3.87(\mathrm{~s}, 3 \mathrm{H}$, Ar-OCH $\left.H_{3}\right), 3.17(\mathrm{~s}, 1 \mathrm{H},-\mathrm{C} \equiv \mathrm{CH}), 2.00\left(\mathrm{~m}, 4 \mathrm{H},-\mathrm{CH}_{2}\left(\mathrm{CH}_{2}\right)_{6} \mathrm{CH}_{3}\right)$, 1.23-1.08 (m, 20H, $\left.-\mathrm{CH}_{2}\left(\mathrm{CH}_{2}\right)_{5} \mathrm{C}_{2} \mathrm{H}_{5}\right), 0.84(\mathrm{t}, J=7.2 \mathrm{~Hz}, 6 \mathrm{H}$, $\left.-\left(\mathrm{CH}_{2}\right)_{7} \mathrm{CH}_{3}\right), 0.65\left(\mathrm{~m}, 4 \mathrm{H},-\left(\mathrm{CH}_{2}\right)_{6} \mathrm{CH}_{2} \mathrm{CH}_{3}\right) .{ }^{13} \mathrm{C} \mathrm{NMR}(75 \mathrm{MHz}$, $\left.\mathrm{CDCl}_{3}\right), \delta$ (ppm): 159.4, 151.7, 151.0, 141.6, 140.0, 137.0, 136.9, 136.4, 131.2, 130.2, 128.8, 128.2, 128.0, 127.7, 126.8, 126.6, 126.5, $126.2,125.7,120.7,120.3,120.1,119.6,114.2(\mathrm{Ar}$ and $-\mathrm{CH}=\mathrm{CH}-$ ), $84.8(-C \equiv \mathrm{CH}) ; 77.2(-\mathrm{C} \equiv C \mathrm{H}), 55.3\left(\mathrm{Ar}^{-} \mathrm{OCH}_{3}\right), 55.1,40.4,31.8$, 30.0, 29.2, 23.7, 22.6, $14.1\left(-\left(\mathrm{CH}_{2}\right)_{7} \mathrm{CH}_{3}\right)$. Anal. calcd for $\mathrm{C}_{48} \mathrm{H}_{56} \mathrm{O}$ : C 88.84, H 8.70. Found: C 88.80, H 8.64.

2-Ethynyl-7-(4-(4-methylstyryl)styryl)-9,9-dioctyl-9H-fluorene (M4). This compound was prepared as the similar approach of M1, using $\mathbf{4 c}$ instead of 3, as the starting material. Product was recrystallized from dichloromethane/ethanol mixture $(1: 1 \mathrm{v} / \mathrm{v})$, 
and obtained as a yellow powder. Yield: $67 \%$. Mp: $144.2{ }^{\circ} \mathrm{C}$. IR (KBr), $\nu\left(\mathrm{cm}^{-1}\right): 3316$ (三C-H), 3022, 963 (=C-H), 2926, 2852 $\left(\mathrm{CH}_{2}, \mathrm{CH}_{3}\right), 2106(\mathrm{C} \equiv \mathrm{C}), 1514,1464,825$ (Ar). ${ }^{1} \mathrm{H}$ NMR (400 $\mathrm{MHz}, \mathrm{CDCl}_{3}$ ), $\delta$ (TMS, ppm): 7.70-7.64 (q, $\left.J=8.0 \mathrm{~Hz}, 2 \mathrm{H}\right), 7.58-$ $7.44(\mathrm{~m}, 10 \mathrm{H}), 7.26-7.07(\mathrm{~m}, 6 \mathrm{H}), 3.17(\mathrm{~s}, 1 \mathrm{H},-\mathrm{C} \equiv \mathrm{CH}), 2.39$ (s, $\left.3 \mathrm{H}, \mathrm{Ar}-\mathrm{CH}_{3}\right), 2.01\left(\mathrm{~m}, 4 \mathrm{H},-\mathrm{CH}_{2}\left(\mathrm{CH}_{2}\right)_{6} \mathrm{CH}_{3}\right), 1.23-1.08(\mathrm{~m}, 20 \mathrm{H}$, $\left.-\mathrm{CH}_{2}\left(\mathrm{CH}_{2}\right)_{5} \mathrm{C}_{2} \mathrm{H}_{5}\right), 0.84\left(\mathrm{t}, J=6.8 \mathrm{~Hz}, 6 \mathrm{H},-\left(\mathrm{CH}_{2}\right)_{7} \mathrm{CH}_{3}\right), 0.65(\mathrm{~m}$, $\left.4 \mathrm{H},-\left(\mathrm{CH}_{2}\right)_{6} \mathrm{CH}_{2} \mathrm{CH}_{3}\right) .{ }^{13} \mathrm{C} \mathrm{NMR}\left(75 \mathrm{MHz}, \mathrm{CDCl}_{3}\right), \delta(\mathrm{ppm}): 151.7$, 151.0, 141.6, 140.1, 137.6, 137.0, 136.9, 136.6, 134.6, 131.2, $129.4,128.9,128.6,128.0,127.3,126.8,126.7,126.5,126.4$, $125.8,120.8,120.3,120.2,119.6(\mathrm{Ar}$ and $-\mathrm{CH}=\mathrm{CH}-), 84.8$ $(-C \equiv \mathrm{CH}), 77.2(-\mathrm{C} \equiv \mathrm{CH}), 55.1,40.4,31.8,30.0,29.2,23.7,22.6$, $14.1\left(-\left(\mathrm{CH}_{2}\right)_{7} \mathrm{CH}_{3}\right), 21.3\left(\mathrm{Ar}-\mathrm{CH}_{3}\right)$. Anal. calcd for $\mathrm{C}_{48} \mathrm{H}_{56}$ : C 91.08, H 8.92. Found: C 91.11, H 8.90.

2-Ethynyl-7-(4-(4-methylstyryl)styryl)-9,9-didodecyl-9H-fluorene (M5). This compound was prepared as the similar approach of M1, using $\mathbf{4 d}$ instead of 3, as the starting material. Product was recrystallized from dichloromethane/ethanol mixture $(1: 1 \mathrm{v} / \mathrm{v})$, and obtained as a yellow powder. Yield: 65\%. Mp: $104.8{ }^{\circ} \mathrm{C}$. IR (KBr), $\nu\left(\mathrm{cm}^{-1}\right): 3308$ (三C-H); 3023, 960 (=C-H), 2926, $2851\left(\mathrm{CH}_{2}, \mathrm{CH}_{3}\right), 2104$ (C三C), 1514, 1463, 826 (Ar). ${ }^{1} \mathrm{H}$ NMR (400 MHz, $\left.\mathrm{CDCl}_{3}\right), \delta$ (TMS, ppm): 7.70-7.64 (q, $=$ $8.0 \mathrm{~Hz}, 2 \mathrm{H}) 7.57-7.44(\mathrm{~m}, 10 \mathrm{H}), 7.22-7.11(\mathrm{~m}, 6 \mathrm{H}), 3.17(\mathrm{~s}, 1 \mathrm{H}$, $-\mathrm{C} \equiv \mathrm{CH}), 2.39\left(\mathrm{~s}, 3 \mathrm{H}, \mathrm{Ar}-\mathrm{CH}_{3}\right), 2.00\left(\mathrm{~m}, 4 \mathrm{H},-\mathrm{CH}_{2}\left(\mathrm{CH}_{2}\right)_{10} \mathrm{CH}_{3}\right)$, 1.33-1.01 (m, 36H, $\left.-\mathrm{CH}_{2}\left(\mathrm{CH}_{2}\right)_{9} \mathrm{C}_{2} \mathrm{H}_{5}\right), 0.88(\mathrm{t}, J=6.8 \mathrm{~Hz}, 6 \mathrm{H}$, $\left.-\left(\mathrm{CH}_{2}\right)_{11} \mathrm{CH}_{3}\right), 0.65\left(\mathrm{~m}, 4 \mathrm{H},-\left(\mathrm{CH}_{2}\right)_{10} \mathrm{CH}_{2} \mathrm{CH}_{3}\right) .{ }^{13} \mathrm{C}$ NMR (75 $\mathrm{MHz}, \mathrm{CDCl}_{3}$ ), $\delta$ (ppm): 151.7, 151.0, 141.6, 140.1, 137.6, 137.0, $136.9,136.6,134.6,131.2$, 129.4, 128.9, 128.6, 128.0, 127.3, 126.8, 126.7, 126.5, 126.4, 125.8, 120.8, 120.3, 120.2, 119.6 (Ar and $-\mathrm{CH}=\mathrm{CH}-), 84.8(-C \equiv \mathrm{CH}), 77.2(-\mathrm{C} \equiv C \mathrm{H}), 55.1,40.4,31.9$, 30.0, 29.6, 29.5, 29.3, 29.2, 23.7, 22.6, $14.1\left(-\left(\mathrm{CH}_{2}\right)_{11} \mathrm{CH}_{3}\right), 21.3$ $\left(\mathrm{Ar}-\mathrm{CH}_{3}\right)$. Anal. calcd for $\mathrm{C}_{56} \mathrm{H}_{72}$ : C 90.26, $\mathrm{H}$ 9.74. Found: C 90.19, H 9.80 .

\subsection{Polymerization}

Scheme 2 all the polymerization reactions and manipulations were performed under purified nitrogen using Schlenk techniques either in vacuum-line system or an inert-atmosphere glove box, except for the purification of the polymers, which were done in open air. Typical procedure is given below: $1.0 \mathrm{mmol}$ of the monomer was added into a baked $20 \mathrm{~mL}$ Schlenk tube with a side arm. The tube was evacuated under vacuum and then flushed with dry nitrogen three times through the side arm. Three milliliters of dioxane was injected into the tube to dissolve the monomer. The catalyst solution was prepared in another tube by dissolving $4.6 \mathrm{mg}(0.01 \mathrm{mmol})$ $[\mathrm{Rh}(\mathrm{nbd}) \mathrm{Cl}]_{2}$ and $2.02 \mathrm{mg}(0.02 \mathrm{mmol}) \mathrm{Et}_{3} \mathrm{~N}$ in $2 \mathrm{~mL}$ of dioxane, which was transferred to the monomer solution using a hypodermic syringe. The reaction mixture was stirred at $60^{\circ} \mathrm{C}$ under nitrogen for $4 \mathrm{~h}$. The mixture was then diluted with $5 \mathrm{~mL}$ of dioxane and added dropwise to $200 \mathrm{~mL}$ of methanol under stirring. The precipitate was centrifuged and redissolved in THF. The THF solution was added dropwise into $200 \mathrm{~mL}$ of methanol to precipitate the polymer. The dissolution-precipitation process was repeated three times, and the finally isolated precipitant was dried under vacuum at $30{ }^{\circ} \mathrm{C}$ to a constant weight.

Poly[2-ethynyl-7-(4-nitrostyryl)-9,9-dioctyl-9H-fluorene] (P1). Deep red solid. Yield: 60\%. GPC: $M_{\mathrm{w}}=10100$, PDI $=1.384$ (polystyrene calibration, Table 1, no. 1). IR (KBr), $\nu\left(\mathrm{cm}^{-1}\right): 2927$, $2857\left(\mathrm{CH}_{2}, \mathrm{CH}_{3}\right), 1610,856(\mathrm{Ar}), 1513,1340\left(\mathrm{NO}_{2}\right) .{ }^{1} \mathrm{H}$ NMR (400 $\mathrm{MHz}, \mathrm{CDCl}_{3}$ ), $\delta$ (TMS, ppm): 8.32 (d, 2H), 8.07-6.92 (br, 10H), 5.43 (s, 1H), 1.93 (br, 4H), 1.05 (br, 22H), 0.81 (br, 8H).

Poly[2-ethynyl-7-(4-(4-nitrostyryl)styryl)-9,9-dioctyl-9H-fluorene] (P2). Deep red solid. Yield: 38\%. GPC: $M_{\mathrm{w}}=4910, \mathrm{PDI}=$ 1.259 (polystyrene calibration, Table 1, no. 3). IR (KBr), $\nu\left(\mathrm{cm}^{-1}\right)$ : 3022, 961 (=C-H); 2923, $2851\left(\mathrm{CH}_{2}, \mathrm{CH}_{3}\right), 1584,1466,821(\mathrm{Ar})$, 1511, $1339\left(\mathrm{NO}_{2}\right) .{ }^{1} \mathrm{H}$ NMR (400 $\left.\mathrm{MHz}, \mathrm{CDCl}_{3}\right), \delta$ (TMS, ppm): 8.27 (d, $J=8.4 \mathrm{~Hz}, 2 \mathrm{H}) 7.68-7.54(\mathrm{br}, 12 \mathrm{H}), 7.32-7.17(\mathrm{br}, 4 \mathrm{H})$, 7.02 (s, 1H), 2.07 (br, 4H, $\left.-\mathrm{CH}_{2}\left(\mathrm{CH}_{2}\right)_{6} \mathrm{CH}_{3}\right), 1.28-1.08$ (br, 20H, $\left.{ }_{-} \mathrm{CH}_{2}\left(\mathrm{CH}_{2}\right)_{5} \mathrm{C}_{2} \mathrm{H}_{5}\right)$, 0.91-0.81 (br, $\left.10 \mathrm{H},-\left(\mathrm{CH}_{2}\right)_{6} \mathrm{CH}_{2} \mathrm{CH}_{3}\right) \cdot{ }^{13} \mathrm{C}$ NMR (75 MHz, $\mathrm{CDCl}_{3}$ ), $\delta$ (ppm): 151.7, 151.0, 146.8, 143.9, 141.5-138.1, 136.7, 135.4, 132.9, 131.2, 129.9, 127.6-124.2, 120.9-119.6 ( $\mathrm{Ar}$ and $-\mathrm{CH}=\mathrm{CH}-$ ), 55.1, 40.4, 31.8, 30.0-29.2, 23.7, 22.6, $14.1\left(-\left(\mathrm{CH}_{2}\right)_{7} \mathrm{CH}_{3}\right)$.

Poly[2-ethynyl-7-(4-(4-methoxystyryl)styryl)-9,9-dioctyl-9Hfluorene] (P3). Deep red solid. Yield: 65\%. GPC: $M_{\mathrm{w}}=96200$, PDI $=1.585$ (polystyrene calibration, Table 1, no. 5). IR (KBr), $\nu$ $\left(\mathrm{cm}^{-1}\right)$ : 3021, 958 (=C-H), 2926, $2852\left(\mathrm{CH}_{2}, \mathrm{CH}_{3}\right), 1605,1512$, 1462, 824 (Ar), 1251, 1036 (C-O-C). ${ }^{1} \mathrm{H}$ NMR (400 MHz, $\mathrm{CDCl}_{3}$ ), $\delta$ (TMS, ppm): 7.57-7.49 (br, 12H), 7.26-6.92 (br, 6H), 7.02 (s, $1 \mathrm{H}$ ), 3.87 (s, 3H, Ar-OCH $H_{3}$, 2.07 (br, $\left.4 \mathrm{H},-\mathrm{CH}_{2}\left(\mathrm{CH}_{2}\right)_{6} \mathrm{CH}_{3}\right), 1.28-$ $1.08\left(\mathrm{~m}, 20 \mathrm{H},-\mathrm{CH}_{2}\left(\mathrm{CH}_{2}\right)_{5} \mathrm{C}_{2} \mathrm{H}_{5}\right), 0.91-0.81\left(\mathrm{br}, 10 \mathrm{H},-\left(\mathrm{CH}_{2}\right)_{6}-\right.$ $\left.\mathrm{CH}_{2} \mathrm{CH}_{3}\right) \cdot{ }^{13} \mathrm{C}$ NMR (75 MHz, $\mathrm{CDCl}_{3}$ ), $\delta$ (ppm): 159.4, 151.7, 151.0, 141.6, 140.0, 137.0-136.4, 131.2, 130.2, 128.8-125.7, 120.7, 120.3, 120.1, 119.6, 114.2 (Ar and -CH=CH-), 55.3 (Ar$\left.\mathrm{OCH}_{3}\right) ; \quad 55.1, \quad 40.4, \quad 31.8, \quad 30.0, \quad 29.2, \quad 23.7, \quad 22.6, \quad 14.1$ $\left(-\left(\mathrm{CH}_{2}\right)_{7} \mathrm{CH}_{3}\right)$.

Poly[2-ethynyl-7-(4-(4-methylstyryl)styryl)-9,9-dioctyl-9H-fluorene] (P4). Deep red solid. Yield: 67\%. GPC: $M_{\mathrm{w}}=152000$, $\mathrm{PDI}=2.269$ (polystyrene calibration, Table 1 , no. 6) IR (KBr), $\nu\left(\mathrm{cm}^{-1}\right)$ : 3022, 963 (=C-H), 2926, $2852\left(\mathrm{CH}_{2}, \mathrm{CH}_{3}\right), 1514,1464$, 825 (Ar). ${ }^{1} \mathrm{H}$ NMR (400 MHz, $\mathrm{CDCl}_{3}$ ), $\delta$ (TMS, ppm): 7.55-7.41 (br, 12H), 7.21-7.12 (br, 6H), $7.02(\mathrm{~s}, 1 \mathrm{H}), 2.39$ (s, 3H, Ar-CH $\mathrm{CH}_{3}$, 2.07 (br, $\left.4 \mathrm{H},-\mathrm{CH}_{2}\left(\mathrm{CH}_{2}\right)_{6} \mathrm{CH}_{3}\right), 1.28-1.10\left(\mathrm{br}, 20 \mathrm{H},-\mathrm{CH}_{2}\left(\mathrm{CH}_{2}\right)_{5}-\right.$ $\mathrm{C}_{2} \mathrm{H}_{5}$ ), 0.91-0.81 (br, 10H, $\left.-\left(\mathrm{CH}_{2}\right)_{6} \mathrm{CH}_{2} \mathrm{CH}_{3}\right) .{ }^{13} \mathrm{C}$ NMR $(75 \mathrm{MHz}$, $\mathrm{CDCl}_{3}$ ), $\delta$ (ppm): 151.0, 141.0, 137.5-134.6, 129.4-126.4, 120.6

Table 1 Polymerization summary of P1-P5 ${ }^{a}$

\begin{tabular}{lllll}
\hline Entry & Polymer & Yield $(\%)$ & $M_{\mathrm{w}}{ }^{b}$ & $M_{\mathrm{w}} / M_{\mathrm{n}}{ }^{c}$ \\
\hline 1 & P1 & 60 & 10100 & 1.384 \\
2 & P1 $^{\prime}$ & 67 & 12760 & 2.121 \\
3 & P2 & 38 & 4910 & 1.259 \\
4 & P2 & 54 & 9650 & 2.080 \\
5 & P3 & 65 & 96200 & 1.585 \\
6 & P4 & 67 & 152000 & 2.269 \\
7 & P5 & 84 & 257600 & 1.882
\end{tabular}

${ }^{a}$ Unless otherwise specified, the polymerization was carried out in dioxane under nitrogen with $\left[\mathrm{Rh}(\mathrm{ncb}) \mathrm{Cl}_{2}\right]_{2}-\mathrm{Et}_{3} \mathrm{~N}$ as a catalyst for $4 \mathrm{~h}$ at $60{ }^{\circ} \mathrm{C}$. ${ }^{b}$ Estimated by GPC in term of polystyrene calibration. ${ }^{c}$ Polymerized in THF. 
(Ar and $-\mathrm{CH}=\mathrm{CH}-$ ), 55.0, 40.4, 31.9-29.2, 23.9-22.6, 14.1 $\left(-\left(\mathrm{CH}_{2}\right)_{7} \mathrm{CH}_{3}\right), 21.3\left(\mathrm{Ar}-\mathrm{CH}_{3}\right)$.

Poly[2-ethynyl-7-(4-(4-methylstyryl)styryl)-9,9-didodecyl-9Hfluorene] (P5). Deep red solid. Yield: 84\%. GPC: $M_{\mathrm{w}}=257$ 600, PDI $=1.882$ (polystyrene calibration, Table 1 , no. 7). IR (KBr), $\nu\left(\mathrm{cm}^{-1}\right)$ : 3023, 960 (=C-H); 2926, $2851\left(\mathrm{CH}_{2}, \mathrm{CH}_{3}\right), 1514,1463$, 826 (Ar). ${ }^{1} \mathrm{H}$ NMR (400 MHz, $\mathrm{CDCl}_{3}$ ), $\delta$ (TMS, ppm): 7.56-7.38 (br, 12H), 7.22-7.11 (br, 6H), 7.02 (s, 1H), 2.39 (s, 3H, Ar-CH $\mathrm{CH}_{3}$, 2.07 (br, $\left.4 \mathrm{H}, \quad-\mathrm{CH}_{2}\left(\mathrm{CH}_{2}\right)_{10} \mathrm{CH}_{3}\right), \quad 1.28-1.11$ (br, $36 \mathrm{H}$, $\left.-\mathrm{CH}_{2}\left(\mathrm{CH}_{2}\right)_{9} \mathrm{C}_{2} \mathrm{H}_{5}\right), 0.91-0.81$ (br, $\left.10 \mathrm{H},-\left(\mathrm{CH}_{2}\right)_{10} \mathrm{CH}_{2} \mathrm{CH}_{3}\right) \cdot{ }^{13} \mathrm{C}$ NMR (75 MHz, $\mathrm{CDCl}_{3}$ ), $\delta$ (ppm): 151, 141, 137.5-134.6, 129.4126.4, 120.8-119.6 ( $\mathrm{Ar}$ and $-\mathrm{CH}=\mathrm{CH}-$ ), 55.1, 40.4, 31.9-29.2, 23.7, 22.6, $14.1\left(-\left(\mathrm{CH}_{2}\right)_{11} \mathrm{CH}_{3}\right), 21.3\left(\mathrm{Ar}-\mathrm{CH}_{3}\right)$.

\section{Results and discussion}

\subsection{Synthesis and polymerization}

We designed and synthesized one styryl-substituted fluorene derivate M1 and four different stilbene-substituted fluorene derivates M2-M5 with different terminal substituted group $\left(\mathrm{NO}_{2}, \mathrm{OCH}_{3}\right.$ and $\left.\mathrm{CH}_{3}\right)$ and side alkyl chain (Scheme 1). The Wittig Horner reaction of these different benzaldehyde derivatives with 4-vinylbenzyl chloride gave stilbene derivatives 1a-1c. 2,7-Dibromofluorene were alkylated at the 9 position by 1-bromooctane or 1-bromododecane in strongly basic conditions to form $\mathbf{2 a - 2 b . ~} 3$ was prepared from $2 \mathbf{a}$ and $p$-nitrostyrene by Heck reaction, and then $\mathbf{4 a - 4 d}$ were made from $\mathbf{2 a - 2 b}$ and $\mathbf{1 a - 1 c}$ by the same reaction. The resultant products ( 3 and $\mathbf{4 a - 4 d )}$ were then reacted with 2-methyl-3-butyn-2-ol using $\mathrm{Pd}\left(\mathrm{PPh}_{3}\right)_{2} \mathrm{Cl}_{2}$ as the catalyst, the obtained semifinished products with 2-propyl2-ol group were cleaved in dioxane/ $\mathrm{NaOH}$ solution to give the desirable monomers M1-M5 in high yields, respectively.

All polymerization reactions were carried out under nitrogen atmosphere using a standard Schlenk vacuum-line system. We
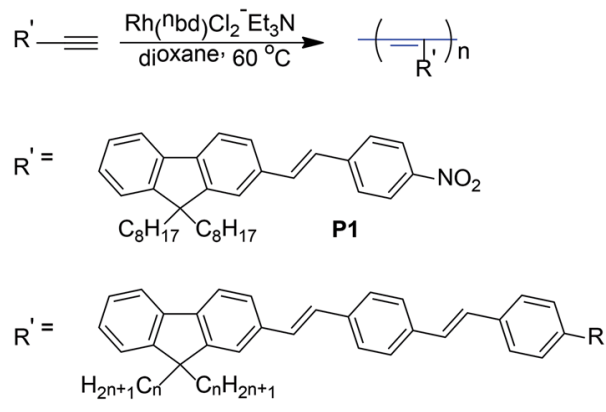

P2: $n=8, R=\mathrm{NO}_{2} ; \mathbf{P 3}: \mathrm{n}={ }^{\prime}, \mathrm{R}=\mathrm{OCH}_{3}$
P4: $\mathrm{n}=\mathrm{8}^{\prime}, \mathrm{R}=\mathrm{CH}_{3} ; \mathbf{P 5}: \mathrm{n}={ }_{12}, \mathrm{R}=\mathrm{CH}_{3}$

Scheme 2 Synthetic routes to the polymers P1-P5.

first attempted to polymerize these monomers (M1-M5) using the classical metathesis catalysts, such as $\mathrm{WCl}_{6}-\mathrm{Ph}_{4} \mathrm{Sn}, \mathrm{TaCl}_{5}-$ $\mathrm{Ph}_{4} \mathrm{Sn}$ and $\mathrm{MoCl}_{5}-\mathrm{Ph}_{4} \mathrm{Sn}$. However, only trace polymeric products were obtained. On the contrary, $[\mathrm{Rh}(\mathrm{nbd}) \mathrm{Cl}]_{2} / \mathrm{Et}_{3} \mathrm{~N}$ catalyst effectively works for these monomers and the results are listed in Table 1. It was found that $\mathbf{P 1}$ and $\mathbf{P 2}$ were partly insoluble in dioxane, only giving molecular weight of the soluble part of the polymers (Table 1, entry 1 and 3). However, the yield of $\mathbf{P} \mathbf{1}^{\prime}$ and P2' was increased by using THF as the polymerization solvent (Table 1, entry 2 and 4). P1 and P2 exhibited the low polymerization yield in dioxane, which may be mainly originated from the enwrapped effect of insoluble polymer to prevent further polymerization of monomers. Moreover, the polymerization yield of $\mathbf{P 2}$ in dioxane or $\mathbf{P 2}{ }^{\prime}$ in THF was almost lower than that of corresponding $\mathbf{P 1}$ or $\mathbf{P 1}{ }^{\prime}$, hinting that polymerization activity of monomer M2 may be lower than that of M1. The result exhibited that polymerization activity of functionalized acetylene monomer decreased with the increase of conjugated chain length of substituted chromophore group. P3, P4, and P5 were
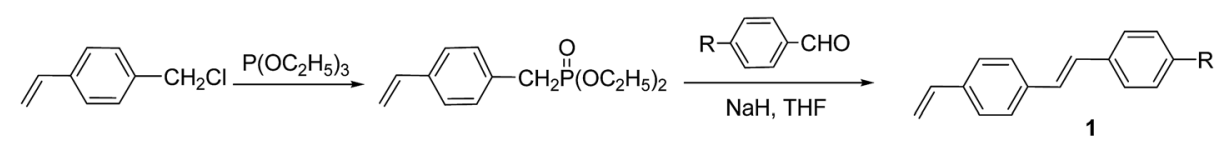

1a: $\mathrm{R}=\mathrm{NO}_{2}, 1 \mathrm{~b}: \mathrm{R}=\mathrm{OCH}_{3}, 1 \mathrm{c}: \mathrm{R}=\mathrm{CH}_{3}$

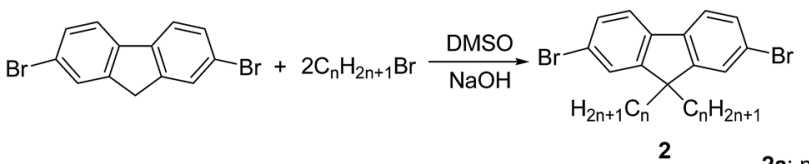

2a: $n=8,2 b: n=12$
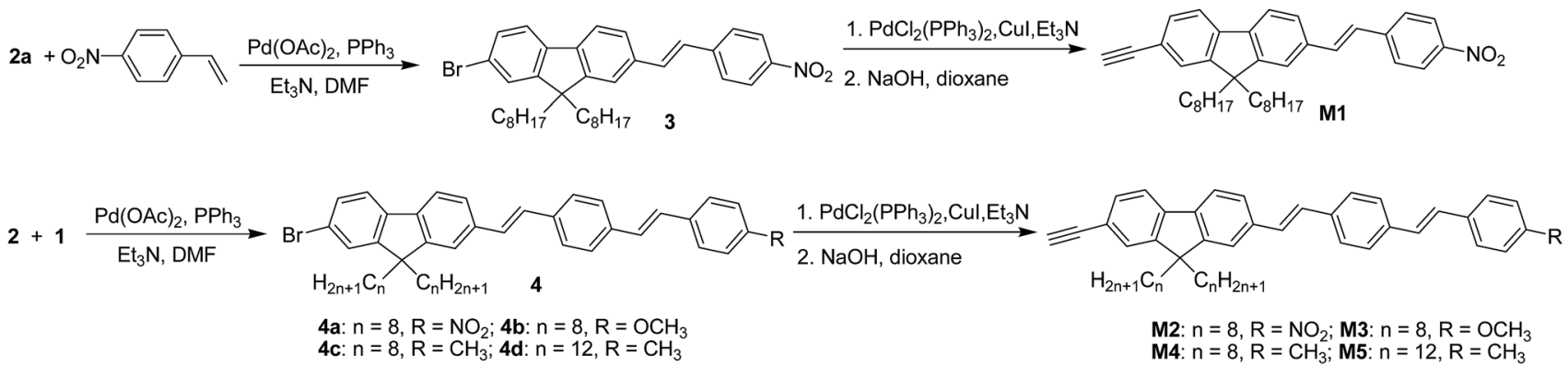

Scheme 1 Synthetic routes of monomer molecules M1-M5. 
soluble in dioxane and gave dark red polymers with high molecular weight $\left(M_{\mathrm{w}}=9 \times 10^{4}-2.5 \times 10^{5}\right)$ in high polymerization yield (Table 1, entry 5, 6, 7), and P5 obtained the highest molecular weight and yield, hinting that the longer flexible alkyl side chain on fluorene was beneficial to polymerization of monomer.

\subsection{Solubility}

It's reported that PAs have weak solubilities in common solvents. ${ }^{25}$ However, when the styryl/stilbene-fluorene pendant with flexible alkyl group as side tailor was incorporated into the PA backbone chain, all the polymers were readily soluble in various common organic solvents such as chloroform, toluene, THF, and 1,2-dichloroethane.

\subsection{Structural characterization of the polymers}

All the polymers and corresponding monomers were characterized by standard spectroscopic methods, from which satisfactory analysis data were obtained (see the Experimental section for details). An example of the FTIR spectrum of P4 is given in Fig. 1. For comparison, the FTIR spectrum of its corresponding monomer $\mathbf{M 4}$ is also given in the same figure. As can be seen from Fig. 1, M4 clearly exhibits two characteristic absorptions at 3319 and $2104 \mathrm{~cm}^{-1}$, respectively, corresponding to the $\equiv \mathrm{C}-\mathrm{H}$ and $\mathrm{C} \equiv \mathrm{C}$ stretching vibrations in the monosubstituted acetylene. The acetylenic absorption bands disappear in the spectrum of $\mathbf{P 4}$ and the characteristic vibration intensity of aromatic ring is significantly enhanced, suggesting that the triple bond in the monomer has changed to double bond in the polymer. Similar results (Fig. S1-S4†) were found in other monomers (M1-M3 and M5) and their polymers (P1-P3 and P5), suggesting that the triple bonds of these monomers have been transferred to double bond polyacetylene backbone.

Fig. 2 shows the ${ }^{1} \mathrm{H}$ NMR spectra of $\mathbf{M 4}$ and $\mathbf{P 4}$ in chloroform-d, respectively. The absorption peak of the acetylene proton in M4 at 3.17 ppm as a singlet peak, completely disappears in the spectrum of its polymer P4. Meanwhile, all the

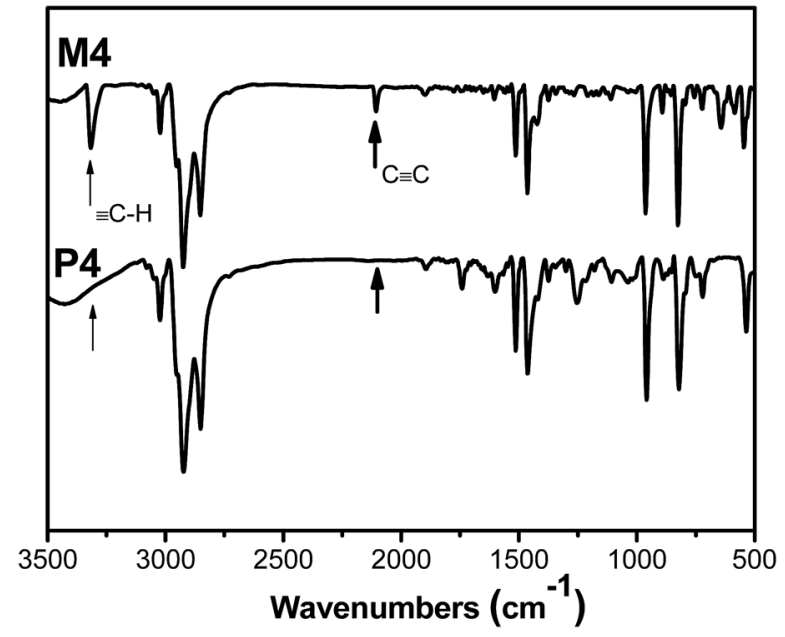

Fig. 1 FTIR spectra of M4 and P4.

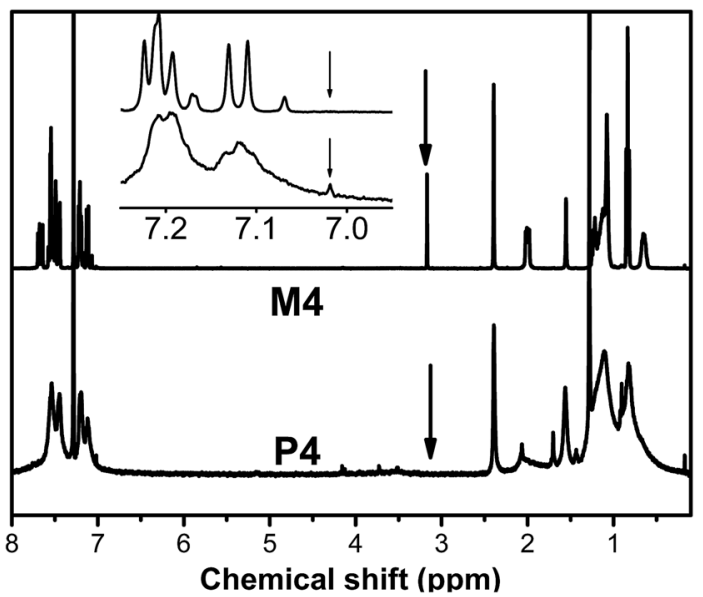

Fig. $2{ }^{1} \mathrm{H}$ NMR spectra of M4 and P4

corresponding characteristic absorption peaks of monomer M4 significantly were widened in the spectra of $\mathbf{P 4}$ with a widened and enhanced peak at $7.02 \mathrm{ppm}$ corresponding to the olefin protons and aromatic protons absorption, further proving that the M4 has been polymerized to yield the functional polyacetylene P4. The results are consistent with that of FTIR spectra. Alternatively, no absorptions are found in the cis-olefin absorption region of 5.20-6.20 ppm, suggesting that these polymers possess a predominantly trans conformation. ${ }^{\mathbf{5 9 6 0}}$ The high trans content may result from the strong steric hindrance of the pendants. ${ }^{61}$

Compared to that of M4, the signals at 77.2 and $84.8 \mathrm{ppm}$ corresponding to the acetylenic carbons disappear in the ${ }^{13} \mathrm{C}$ NMR spectrum of P4 (Fig. S9†), and the relevant absorption peak intensity of the carbon atoms of the aromatic pendants in polymers is significantly enhanced because of the absorption overlapping effect of the olefin carbon atoms of the polyacetylene backbone. Thus, the ${ }^{1} \mathrm{H}$ NMR and ${ }^{13} \mathrm{C}$ NMR spectra data further confirm that the acetylene polymerization has taken place. The similar results were found in polymerization of other monomers. The satisfactory spectral data are given in the ESI (Fig. S10-S12†).

\subsection{Linear optical properties}

The linear optical properties of the polymers and corresponding monomers were investigated by UV-vis absorption and fluorescence in THF solutions at $20^{\circ} \mathrm{C}$ in extremely diluted solution (2 $\times 10^{-5} \mathrm{M}$ ) and these spectra are depicted in Fig. 3. It can be seen from Fig. 3 that the maximum absorption wavelengths of P1-P5 are located at 400, 406, 388, 385 and $385 \mathrm{~nm}$, respectively, which are assigned to the $\pi-\pi^{*}$ electronic transitions of the corresponding conjugated styryl/stilbene-fluorene chromophores. Obviously, the maximum absorption wavelengths of the polymers changes at the different terminal substituents or the length of conjugated systems, but aren't influenced by the length of alkyl flexible chain. The absorption band of the nitrosubstituted polymers P1 and P2 shows significantly red-shifts, which results from the larger $\pi$-electron delocalization and 

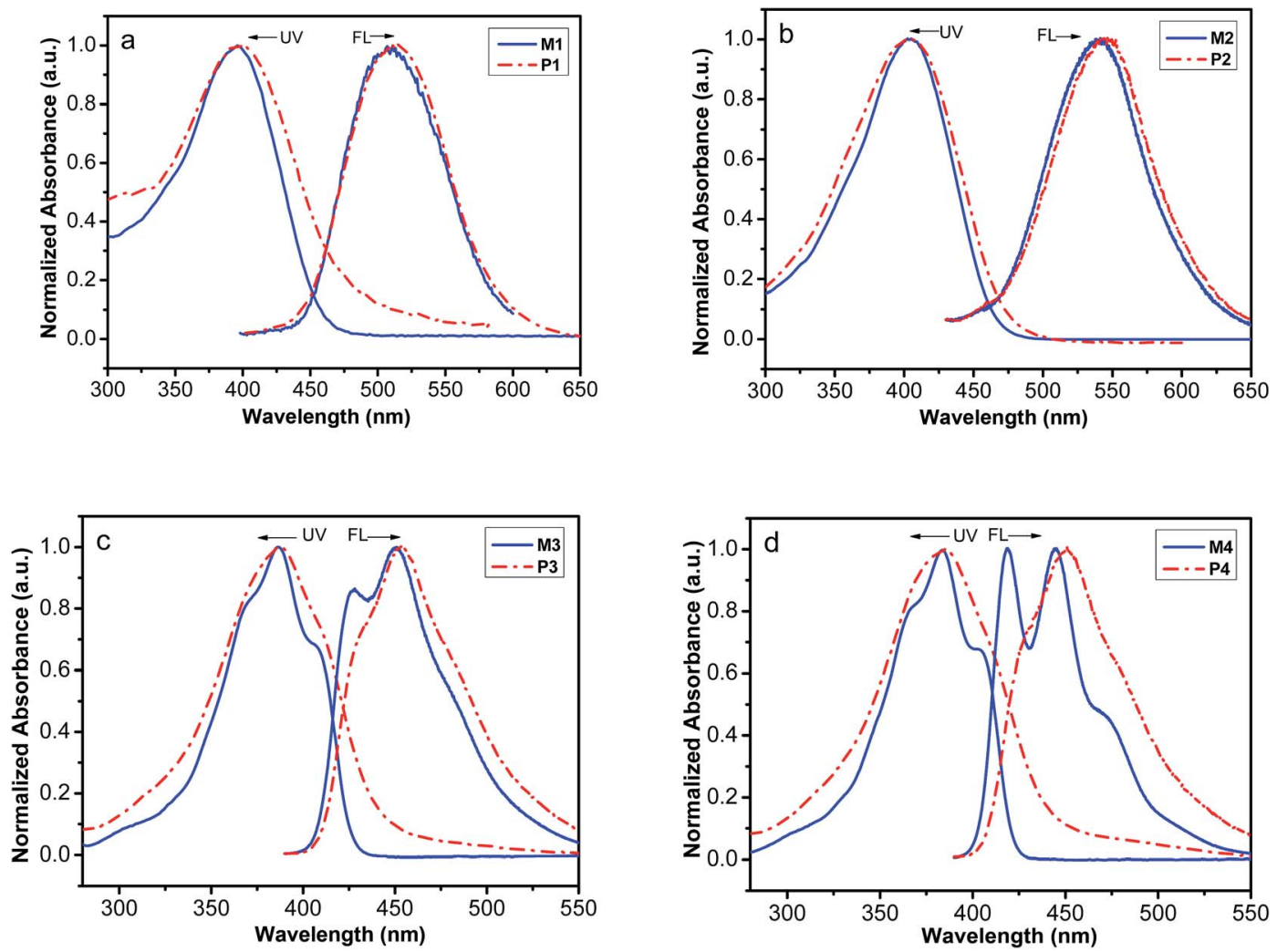

Fig. 3 Normalized UV-vis absorption and FL emission spectra of the monomers (solid line) and corresponding polymers (dash dot) in THF solutions (spectra of M5 and P5 were showed in Fig. S13†).

stronger dipolar effect of $\mathrm{NO}_{2}$ group. P2 with longer conjugated chromophore substituent shows larger red-shift at maximum absorption wavelength than P1. The absorption band of the methyl-substituted polymers $\mathbf{P 4}$ and $\mathbf{P 5}$ with the different alkyl flexible chain exhibit the almost same maximum absorption wavelength. Although the polymers and their corresponding monomers show nearly the same $\lambda_{\max }$, the polymers show significant broad backbone absorption at longer wavelength than $420 \mathrm{~nm}$, which result from the double contribution of conjugated double-bond backbones of polyacetylene and enhancement conjugation effect between the double-bond backbones of the polyacetylenes and styryl/stilbene-fluorene pendant groups. These results further support that the monomers have been converted to polymers.

An interesting feature for the polymers P1-P5 is that they all possess luminescent properties upon photo irradiation while PA itself is nonluminescent. ${ }^{62}$ When excited at relative maximum absorption wavelength in THF solution, P1-P5 emit fluorescence with maximum emitting peak at around $515 \mathrm{~nm}$, $541 \mathrm{~nm}, 453 \mathrm{~nm}, 450 \mathrm{~nm}$ and $447 \mathrm{~nm}$ respectively, which are higher than that of the corresponding monomers (Fig. 3). The red-shift of fluorescence emitting peak probably results from a strong pendant-pendant intermolecular interaction and/or intramolecular interaction between styryl/stilbene-fluorene pendants and the polyacetylene conjugation main chain. Similar phenomena are found by Balcar. ${ }^{63}$

\subsection{Optical limiting properties and mechanism}

Many optical limiting materials for visible optical region have been investigated. However, the optical limiting materials for the near-infrared optical region were rarely reported. Thus, we investigated the near infrared optical limiting properties of resultant polymers by a mode-locked Ti:sapphire laser (Quantronix, IMRA) at $780 \mathrm{~nm}$ using 450 femtosecond laser pulses at 1 $\mathrm{kHz}$ repetition rate. For avoiding the influence from thermally induced scattering effects, the optical limiting properties of the polymers in THF solution were measured at low unit concentration $(1 \mathrm{mM})$. Fig. 4 shows the optical limiting properties of P1-P5 in THF and the results are summarized in Table 1S. $\dagger$ For comparison, the optical limiting property of polyphenylacetylene (PPA) at the same condition is also made. The results were shown in Fig. 4. At low incident irradiance, the transmittances of all the polymer solutions remain unchanged with the incident irradiance obeying the Beer-Lambert law. However, the transmittances of the solutions start to deviate from the linear transmission with a further increase in the incident irradiance, and a nonlinear relationship is observed between the output and input irradiance, while that of PPA still rises linearly because of the laser-induced photolysis of the polyacetylene chains, ${ }^{68}$ suggesting that the incorporation of conjugated styryl/stilbene-fluorene group into polyacetylene backbone has endowed the resultant polyacetylenes with novel laser protection characteristics at $780 \mathrm{~nm}$ for 450 femtosecond pulses laser. It is also found that the limiting thresholds of the 


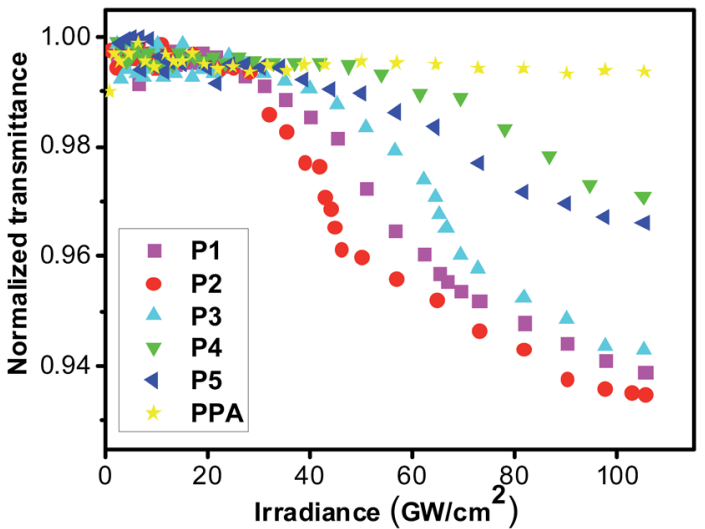

Fig. 4 Optical limiting properties of P1-P5, and polyphenylacetylene (PPA) for femtosecond laser pulse.

resultant polymers, which are defined as the incident irradiance fluence where the transmittance starts to deviate from linearity, are significantly influenced by molecular structures. As seen in Table $1 \mathrm{~S}, \dagger$ polymer P5 $\left(34.9 \mathrm{GW} \mathrm{cm}^{-2}\right)$ with longer tail alkyl chain length in side position of fluorene pendant exhibits better optical limiting properties than $\mathbf{P 4}\left(47.6 \mathrm{GW} \mathrm{cm}^{-2}\right)$. Moreover, polymer $\mathbf{P} 3$ shows the better optical property when the terminal methyl group of stilbene-fluorene pendant of $\mathbf{P 4}$ is replaced by methoxy group. The limiting threshold of $\mathbf{P 2}\left(16.7 \mathrm{GW} \mathrm{cm}^{-2}\right)$ is better than P1 (21.4 $\left.\mathrm{GW} \mathrm{cm}^{-2}\right)$, which should be attributed to the larger electron conjugation of chromophoric moiety in P2. It can be found that $\mathbf{P} 2$ shows the best optical limiting properties (lowest optical limiting thresholds) among all the polymers, owing to the longer conjugated chromophore substituent and larger dipolar effect of strong electron acceptor $\mathrm{NO}_{2}$.

The optical limiting mechanisms of organic compounds are often based on TPA or RSA. Generally, TPA based optical limiting effect can be yielded in principle under the laser irradiation of picosecond or shorter pulses, while RSA is achieved on a nanosecond or longer time scale, owing to the different excited state lifetimes involved in a multilevel energy process. ${ }^{64}$ In this work, the polymers are excited by 450 femtosecond laser pulses at $780 \mathrm{~nm}$. Therefore, we consider that the optical limiting properties of the polymers may mainly originate from TPA.

For investigation of optical limiting mechanism, we performed open-aperture Z-scans with the same laser. The Z-scan experiments of the polymer solution were carried out at $780 \mathrm{~nm}$ using femtosecond laser pulses (450 fs, $1 \mathrm{kHZ}$ ). In theory, the normalized transmittance for the open aperture can be written $a^{65}$

$$
T(z, s=1)=\sum_{m=0}^{\infty} \frac{\left[-q_{0}(z)\right]^{m}}{(m+1)^{3 / 2}}, \quad \text { for }\left|q_{0}\right|<1
$$

where $q_{0}(z)=\alpha_{2} I_{00}(t) L_{\text {eff }} /\left(1+z^{2} / z_{0}^{2}\right)$ with $\beta$ is the TPA coefficient, $I_{0}$ is the intensity of laser beam at focus $(z=0), L_{\text {eff }}=[1-$ $\left.\exp \left(-\alpha_{0} L\right)\right] / \alpha_{0}$ is the effective thickness $(\alpha$ is the linear absorption coefficient, $L$ is the sample thickness, $z_{0}$ is the Rayleigh range of the laser beam, and $z$ is the sample position). The TPA cross-section $\sigma_{2}$ is calculated by ${ }^{55}$

$$
\sigma_{2}=h \nu \beta / N
$$

where $N$ is the number of molecules per $\mathrm{cm}^{3}$ and $h \nu$ is the excitation photon energy. The TPA cross-section $\sigma_{2}$ is expressed in Göppert Mayer (GM) units, in which $1 \mathrm{GM}=1 \times 10^{-50} \mathrm{~cm}^{4} \mathrm{~s}$ per photon. The Z-scan data (or the transmittance as a function of $z$ position) were normalized to the linear (small-signal) transmittance. The nonlinear-optical signal from the solvent was negligible, compared to the signals from the fullerene derivatives. The Z-scan results confirmed that the samples had surprisingly good photostability, which was verified by indifference between the linear absorption spectra measured before and after intense laser irradiation in the Z-scans. In addition, the TPA coefficients $\beta$, could be extracted from the best fitting between the Z-scan theory and the data, and the TPA cross sections were then calculated from the definition (2).

As thermally induced scattering effects and exited-states absorption caused by high irradiance may influence the accuracy of overall TPA cross sections measured, we undertook the Z-scans of P1-P5 in THF with different irradiances. As an example, the results of $\mathbf{P 2}$ are shown in Fig. 5. After calculation from definition (2), it is found that the TPA cross sections $\left(\sigma_{2}\right)$ of the polymers are independent of laser irradiance and remain nearly constant across the irradiance range of interest, indicating the observed nonlinear absorption is induced from a pure third-order nonlinear process (Table 1S $\dagger$ ). Other nonlinear mechanisms such as thermally induced scattering and excited-state absorption, in particular singlet excited-state absorption, are negligible in our experiments. The similar phenomena (Fig. S14-S17†) were also observed in other polymers. Furthermore, it is found that TPA cross sections of the polymers P1-P5 are obviously larger than those of PPA..$^{42}$ Thus, the attachment of styryl/stilbene-fluorene moieties into the PA main chain has endowed the resultant polyacetylenes with enhanced the nonlinear optical properties significantly, which may be attributed to the enhanced conjugation effect between substituent fluorene pendants and conjugated backbone of polyacetylene. The similar results were also found in oxadiazole-

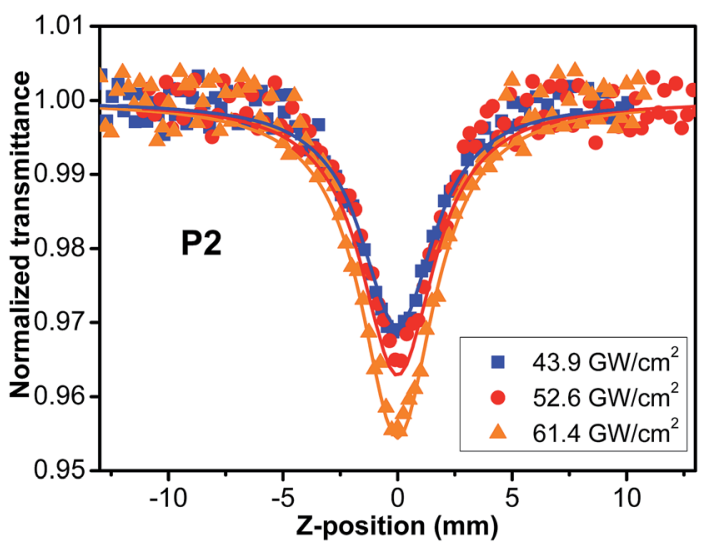

Fig. 5 Open-aperture Z-scans of P2 at the different pulse energy in THF solution. Solid lines are the theoretical fit for two-photon absorption. 
based or azobenzene-based chromophore functionalized polyacetylenes. ${ }^{\mathbf{4 5 , 4 9}}$

Fig. 6 shows the TPF emission spectra of all polymers (P1-P5) in solid form. The maximum TPF peaks of P1-P5 located at $562 \mathrm{~nm}, 584 \mathrm{~nm}, 512 \mathrm{~nm}, 506 \mathrm{~nm}$ and $506 \mathrm{~nm}$, respectively. Comparing the TPF curves of P1-P5 with those of one-photon fluorescence of P1-P5 in THF (Fig. 3), red shifts can be identified, supporting that the re-absorption effects are involved in the nonlinear process in the solid polymers. It is well known that the fluorescence emission intensity presented a square dependence of the fluorescence on the input intensity to prove the TPA nature. When the sample is represented by TPA without stimulated emission, self-quenching, or any dark states influencing the emission intensity, the signal intensity $\left(I_{\text {fluor }}\right)$ of the detected fluorescence from the sample cell is given by ${ }^{55}$

$$
I_{\text {fluor }} \propto I_{0}^{2} \beta l_{0}
$$

here $I_{0}$ is the input light intensity, $l_{0}$ and $\beta$ are the optical path length and TPA coefficient of the medium, respectively. Therefore, Fig. 6(a) and (b) showed the two-photon fluorescence (TPF) spectra at different input light intensities and the log-log plots of the signal intensity $\left(I_{\text {fluor }}\right)$ of the detected fluorescence $v s$. the input light intensity $\left(I_{0}\right)$ of P1-P5, respectively. The results displayed that the log-log plots of the detected TPF intensity are linear with the input light intensity, and the slopes are approximately 2, further confirming that the optical limiting properties are mainly attributed to the molecular TPA absorption of resultant polyacetylenes. ${ }^{66}$

\subsection{Thermal property}

The thermal stability of the resulting polymers was evaluated by thermogravimetric analysis (TGA) under nitrogen atmosphere. It is well known that poly(1-alkyne)s such as poly(1-butyne) and poly(1-hexyne), are so unstable that even the isolation process of the polymer products from the polymerization reactions leads to degradation. ${ }^{59,62}$ However, as shown in Fig. 7, all the fluorenecontaining polyacetylenes exhibit good thermal stability. $T_{\mathrm{d}}$ (defined as the temperature of 5\% weight loss) for P1 is as high as $357^{\circ} \mathrm{C}$, while $T_{\mathrm{d}}$ for $\mathbf{P} 2$ with longer conjugated pendant is at $327{ }^{\circ} \mathrm{C}$. When para-position of stilbene-fluorene substituted by different groups, the resulting polymers showed $T_{\mathrm{d}}$ at 407,397

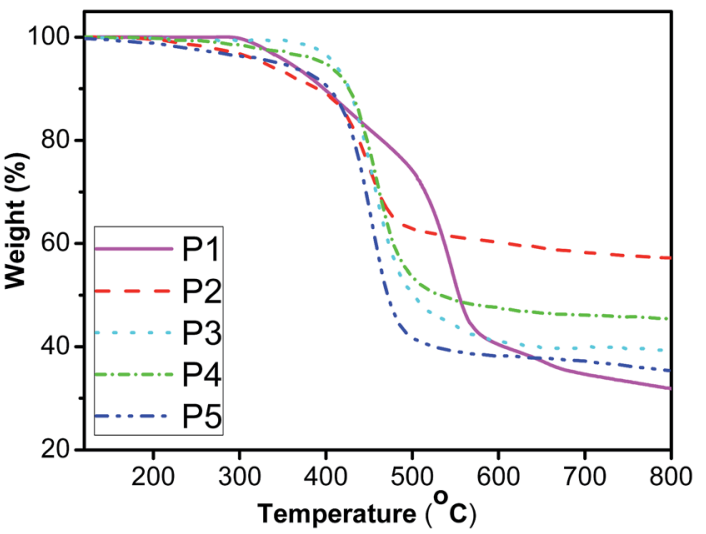

Fig. 7 TGA thermograms of P1-P5.

and $345{ }^{\circ} \mathrm{C}$ for P3, P4 and P5, respectively, which are much higher than that for PPA $\left(225^{\circ} \mathrm{C}\right) . .^{55}$ Thus, the incorporation of the rigid styryl/stilbene-fluorene groups into polyacetylenes significantly improves the thermal stability of resultant polyacetylenes. The enhancement of the thermal stability may be due to the "jacket effect" of the styryl/stilbene-fluorene pendants. Namely, the alternating-double-bond backbone of the polymers may be surrounded by a rigid "jacket" formed through the strong intra- and interchain molecular electronic interaction of the styryl/stilbene-fluorene groups, shielding the polymer main chains from the thermal attack. Similar phenomena were also found by Tang, ${ }^{59,62}$ Masuda $^{67}$ and our previous work. ${ }^{45-48,68,69}$

\section{Conclusions}

In summary, a series of fluorene-containing functionalized polyacetylenes with different molecular structure was successfully prepared using $[\mathrm{Rh}(\mathrm{nbd}) \mathrm{Cl}]_{2}-\mathrm{Et}_{3} \mathrm{~N}$ as the catalyst. The incorporation of conjugated styryl/stilbene-fluorene group into polyacetylene backbone has endowed the resultant polyacetylenes with novel near-infrared laser protection. The optical limiting properties for near infrared laser protection are influenced by their molecular structures. The functional polyacetylene with longer conjugation structure and stronger
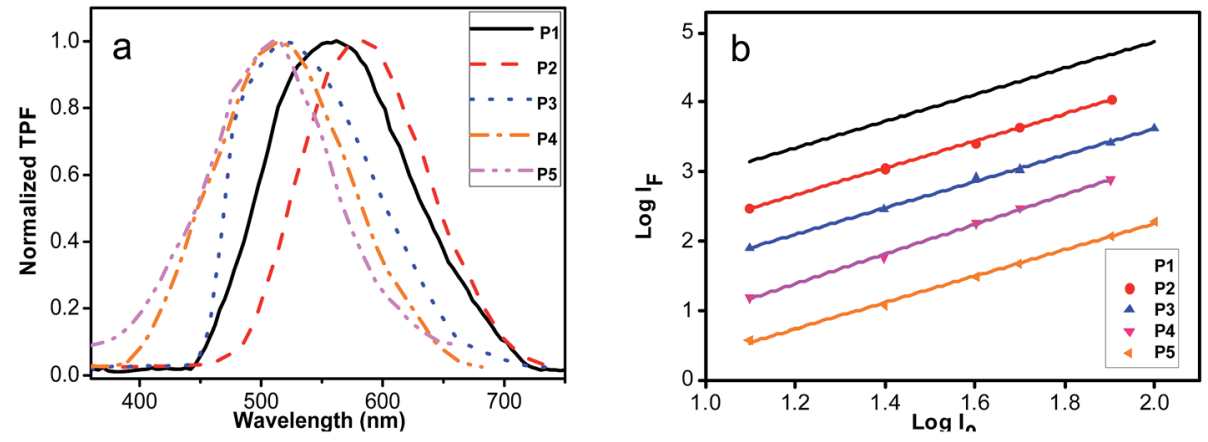

Fig. 6 (a) Two-photon fluorescence (TPF) spectra of P1-P5; (b) the log-log plots of the signal intensity $\left(I_{\text {fluor }}\right)$ of the detected fluorescence vs. the input light intensity $\left(I_{0}\right)$ of P1-P5. 
terminal acceptor $\mathrm{NO}_{2}$ group exhibits better optical limiting properties. The investigation of optical limiting mechanism shows that the optical limiting properties are mainly originated from two-photon absorption of the polymeric molecules. In addition, the thermal stabilities of the resultant polymers are significantly improved due to "jacket effect" resulting from strong intra and inter molecular electronic interaction between polyacetylenes main chain and substituted chromophoric pendants. This work provides an important foundation for design of new materials for preparing soluble functional polyacetylenes with near-infrared laser protection and high thermal properties.

\section{Conflicts of interest}

There are no conflicts to declare.

\section{Acknowledgements}

This research was financially supported by the National Natural Science Fund of China (Grant No. 21671037, 21471030, and 21271040).

\section{Notes and references}

1 Y. L. Du, K. Zhu, Y. Fang, S. L. Zhang, X. R. Zhang and Y. N. Lu, $R S C A d v .$, 2015, 5, 48311-48322.

2 G.-K. Lim, R. G. S. Goh, Z.-L. Chen, J. Clark, W.-H. Ng and L.-L. Chua, Nat. Photonics, 2011, 5, 554-560.

3 M. Feng, H. Zhan and Y. Chen, Appl. Phys. Lett., 2010, 96, 033107.

4 J. Wang, Y. Hernandez, M. Lotya, J. N. Coleman and W. J. Blau, Adv. Mater., 2009, 21, 2430.

5 Y. Chen, Y. Lin, Y. Liu, J. Doyle, N. He, X. Zhuang and W. J. Blau, J. Nanosci. Nanotechnol., 2007, 7, 1268-1283.

6 S. Rahman, S. Mirza, A. Sarkar and G. W. Rayfield, J. Nanosci. Nanotechnol., 2010, 10, 4805-4823.

7 Y. X. Liu, P. Zhang, C. J. He, X. F. Duan, Y. Yu and J. Q. Yao, Acta Phys. Sin., 2005, 54, 3661-3664.

8 J. Wang, Y. Chen, R. Li, Y. Chen, R. Li and W. J. Blau, J. Inorg. Organomet. Polym., 2011, 21, 736-746.

9 J. Wang, Y. Chen and W. J. Blau, J. Mater. Chem., 2009, 19, 7425-7443.

10 R. K. Rekha and A. Ramalingam, J. Mod. Opt., 2009, 56, 10961102.

11 J. Gao, Q. Li, H. Zhao, L. Li, C. Liu and L. Qi, Chem. Mater., 2008, 20, 6263-6269.

12 X. Yu, C. Cao, H. Zhu, Q. S. Li, C. L. Liu and Q. H. Gong, Adv. Funct. Mater., 2007, 17, 1397-1401.

13 C. H. Li, Z. M. Huo and H. P. Zeng, Chem. Res. Chin. Univ., 2010, 31, 1554-1559.

14 S. H. Chi, J. M. Hales, M. Cozzuol, C. Ochoa, M. Fitzpatrick and J. W. Perry, Opt. Express, 2009, 17, 22062-22072.

15 S. M. O'Flaherty, S. V. Hold, T. Torres, Y. Chen, M. J. Cook, M. Hanack and W. J. Blau, Adv. Mater., 2003, 15, 19-20.

16 M. Calvete, G. Y. Yang and M. Hanack, Synth. Met., 2004, 141, 231-243.
17 M. O. Senge, M. Zawadzka, E. G. Notaras, W. J. Blau, M. Fazekas, O. B. Locos and E. M. Ni Mhuircheartaigh, Adv. Mater., 2007, 19, 2737-2774.

18 J. Perry, I.-Y. Lee, K. Mansour, P. Bedworth, X.-L. Wu and T. Wada, Science, 1996, 273, 1533-1536.

19 G. J. Zhou, W. Y. Wong, S. Y. Poon, C. Ye and Z. Lin, Adv. Funct. Mater., 2009, 19, 531-544.

20 G. J. Zhou, W. Y. Wong, C. Ye and Z. Lin, Adv. Funct. Mater., 2007, 17, 963-975.

21 P. F. Zhang, J. Nonlinear Opt. Phys. Mater., 2011, 20, 229236.

22 J. C. Bischof and Z. Qin, Chem. Soc. Rev., 2012, 41, 11911217.

23 D. Chateau, F. Chaput, G. Berginc, O. Maury, C. Andraud and S. Parola, J. Mater. Chem. C, 2014, 2, 5105-5110.

24 Y. Xiong, L. Yan, J. Si, W. Ding, X. Liu, F. Chen and X. Hou, J. Appl. Phys., 2014, 115, 083111-083112.

25 N. Liaros, S. Couris and E. Koudoumas, Appl. Phys. Lett., 2014, 104, 19112-19113.

26 Z. Yang, A. D. Xia, J. S. Ma, Q. S. Li, Z. K. Wu, C. L. Liu and Q. H. Gong, Appl. Phys. Lett., 2005, 86, 061903-061904.

27 S. Husaini, J. M. Murray, L. P. Gonzalez and R. G. Bedford, Appl. Phys. Lett., 2013, 102, 191112-191113.

28 J. Staromlynska, T. J. McKay and P. Wilson, J. Appl. Phys., 2000, 88, 1726-1732.

29 Q. S. Li, Z. G. Liu, C. L. Liu and Q. H. Gong, Opt. Express, 2005, 13, 1833-1838.

30 J. S. Shirk, H. Heckmann, S. R. Flom and M. Hanack, J. Phys. Chem. A, 2000, 104, 1438-1449.

31 C. Li, C. L. Liu, Q. S. Li and Q. H. Gong, Chem. Phys. Lett., 2004, 400, 569-572.

32 M. Cha, A. J. Heeger, J. C. Hummelen, N. S. Sariciftci and F. Wudl, Appl. Phys. Lett., 1995, 67, 3850-3852.

33 G.-J. Zhou and W.-Y. Wong, Chem. Soc. Rev., 2011, 40, 25412566.

34 G.-J. Zhou, W.-Y. Wong, D. Cui and C. Ye, Chem. Mater., 2005, 17, 5209-5217.

35 N. Liaros, E. Koudoumas and S. Couris, Appl. Phys. Lett., 2014, 104, 19112.

36 D. Chateau, Q. Bellier, F. Chaput, P. Feneyrou, G. Berginc, O. Maury, C. Andraud and S. Parola, J. Mater. Chem. C, 2014, 2, 5105-5110.

37 S. Husaini, J. E. Slagle, J. M. Murray, S. Guha, L. P. Gonzalez and R. G. Bedford, Appl. Phys. Lett., 2013, 102, 191112.

38 Z. Yang, Z. K. Wu, J. S. Ma, A. D. Xia, Q. S. Li, C. L. Liu and Q. H. Gong, Appl. Phys. Lett., 2005, 86, 061903.

39 Z. G. Xiao, Y. F. Shi, R. Sun, J. F. Ge, Z. G. Li and Y. L. Song, J. Mater. Chem. C, 2016, 4, 4647-4653.

40 W. S. Fann, S. Benson, J. M. Madey, S. Etemad, G. L. Baker and F. Kajar, Phys. Rev. Lett., 1989, 62, 1492-1495.

41 S. Masashi, S. Fumio and M. Toshio, Polym. Chem., 2011, 2, 1044-1058.

42 J. Z. Liu, J. W. Y. Lam and B. Z. Tang, Chem. Rev., 2009, 109, 5799-5867.

43 Z. Q. Yan, Y. F. Chen, S. Y. Guang, H. Y. Xu and L. F. Li, Polym. Sci., Ser. B, 2011, 53, 535-539. 
44 X. Wang, Y. Yan, T. Liu, X. Y. Su, L. W. Qian and H. Y. Xu, J. Polym. Sci., Part A: Polym. Chem., 2010, 48, 5498-5504.

45 X. Wang, J. C. Wu, P. Wang and B. Z. Tang, J. Polym. Sci., Part A: Polym. Chem., 2008, 46, 2072-2083.

46 X. Y. Su, N. B. Lin and Y. L. Song, Polymer, 2008, 49, 37223730.

47 X. Wang, S. Y. Guang, X. Y. Su and X. Y. Liu, J. Mater. Chem., 2008, 18, 4204-4209.

48 X. Y. Su, J. Y. Yang and X. Y. Liu, J. Polym. Sci., Part A: Polym. Chem., 2008, 46, 4529-4541.

49 S. C. Yin, H. Y. Xu, X. Y. Su, L. Wu, Y. L. Song and B. Z. Tang, Dyes Pigm., 2007, 75, 675-680.

50 S. Y. Guang, S. C. Yin, W. J. Zhu, Y. C. Gao and Y. L. Song, Dyes Pigm., 2007, 73, 285-291.

51 S. C. Yin, Y. C. Gao, Y. L. Song and B. Z. Tang, Dyes Pigm., 2007, 72, 119-123.

52 A. Shukla, Chem. Phys., 2004, 300, 177-188.

53 C. W. Li, K. Yang and H. Y. Xu, J. Phys. Chem. B, 2009, 113, 15730-15733.

54 G. S. He, L. S. Tan, Q. Zheng and P. N. Prasad, Chem. Rev., 2008, 108, 1245-1330.

55 Q. Zheng, G. S. He and P. N. Prasad, Chem. Mater., 2005, 17, 6004-6011.

56 X. H. Ouyang, H. P. Zeng and W. Ji, J. Phys. Chem. B, 2009, 113, 14565-14573.
57 F. G. Haj, B. G. Neel and P. I. H. Bastiaens, Science, 2002, 295, 1708-1711.

58 B. Z. Tang, W. Y. Lam and H. S. Kwok, Macromolecules, 1998, 31, 2419-2432.

59 Y. Fujita, Y. Misumi and M. Tabata, J. Polym. Sci., Part A: Polym. Chem., 1998, 36, 3157-3163.

60 H. Goto, X. M. Dai, T. Ueoka and K. Akagi, Macromolecules, 2004, 37, 4783-4793.

61 J. W. Y. Lam and B. Z. Tang, Acc. Chem. Res., 2005, 38, 745754.

62 H. Balcar, J. Sedlacek and J. Vohlidal, Polymer, 2001, 42, 6709-6721.

63 B. Z. Tang and H. Y. Xu, Macromolecules, 1999, 32, 25692576.

64 B. Gu, S. M. Dharmaprakash and H. T. Wang, Appl. Phys. Lett., 2008, 92, 091118-091121.

65 M. S. Bahae, A. A. Said, T. H. Wei, D. J. Hagan and E. W. V. Stryland, IEEE J. Quantum Electron., 1990, 26, 760769.

66 N. Lin, X. Y. Liu and W. Ji, Adv. Funct. Mater., 2012, 22, 361368.

67 M. Teraguchi and T. Masuda, Macromolecules, 2000, 33, 240242.

68 Z. Yan, H. Xu and X. Y. Liu, Adv. Funct. Mater., 2012, 22, 345352.

69 F. Y. Zhang, J. Li and H. Y. Xu, RSC Adv., 2016, 6, 448-455. 